\title{
Los diagramas de equilibrio de fases como una herramienta para el diseño y comprensión del comportamiento en servicio de los materiales refractarios
}

\author{
A. H. DE AZA, P. PENA, A. CABAllero Y S. DE AZA \\ Instituto de Cerámica y Vidrio (ICV), CSIC. C/ Kelsen 5. 28049 Madrid. España.
}

\begin{abstract}
La aplicación de los diagramas de equilibrio de fases para diseñar materiales refractarios y también para comprender su comportamiento en servicio, ha sido, a lo largo del tiempo, un tema de controversia en el campo de la cerámica y de los refractarios. Sin embargo, en la tecnología refractaria moderna es posible obtener mezclas homogéneas a través de rutas sofisticadas de procesamiento. Como resultado, las velocidades de reacción son generalmente lo suficientemente altas para alcanzar el equilibrio o cuasi-equilibrio durante la cocción convencional del material así como en su comportamiento en servicio. Por lo tanto, hoy en día, los diagramas de equilibrio son válidos no sólo para determinar la tendencia termodinámica de equilibrio durante la cocción del material refractario, sino también para predecir el estado final de equilibrio y, con una buena comprensión de las relaciones de equilibrio, también es posible conocer el camino a través del cual el sistema refractario evoluciona hacia el estado final durante su aplicación. En consecuencia, los diagramas de equilibrio son una potente herramienta para el diseño de nuevos materiales refractarios, así como para una mejor comprensión de su comportamiento en servicio en condiciones de alta temperatura y agentes corrosivos. En el presente trabajo se pretende mostrar, a través de algunos ejemplos pertinentes, cómo el proceso de fabricación de algunos materiales refractarios tradicionales puede ser explicado y también cómo es posible mejorar y / o diseñar nuevos materiales refractarios mediante el uso de los diagramas de equilibrio de fases apropiados.
\end{abstract}

El presente trabajo recopila y revisa la última conferencia plenaria impartida por el Profesor Salvador De Aza sobre el tema.

Palabras claves: Diagramas de equilibrio de fases, refractarios, sistemas: $\mathrm{SiO}_{2}-\mathrm{CaO} ; \mathrm{SiO}_{2}-\mathrm{MgO} ; \mathrm{SiO}_{2}-\mathrm{CaO}_{-} \mathrm{Al}_{2} \mathrm{O}_{3^{\prime}} \mathrm{MgO}_{8}-\mathrm{CaO}_{-} \mathrm{SiO}-\mathrm{ZrO}_{2}$ y $\mathrm{Al}_{2} \mathrm{O}_{3}-\mathrm{MgO}-\mathrm{CaO}$.

The phase equilibrium diagrams as a tool for the design and use of refractories

Refractories are complex materials used at high temperature, in severely corrosive atmospheres and in contact with aggressive liquids. The high temperatures imply that such systems tend to equilibrium and this is frequently attained during service; at least local equilibrium is achieved. This allows the basic principles of phase diagrams to be used in this technology. Traditionally, refractories have been designed to be close to equilibrium so that in-service changes were restricted. Currently, additions of raw materials are often made that will react in use, in a controlled manner, to give favorable effects under the service conditions. Equilibrium diagrams are valid not only for determining the thermodynamic tendency but also for predicting the final equilibrium state and to know the way through which the material moves into the final state. In this context equilibrium diagrams become a powerful tool for a better understanding of the behavior of refractrories during service. After a general consideration on the importance of phase equilibrium diagrams in this field, criteria for using equilibrium diagrams, as a tool for improving traditional refractories and/or designing advanced or new refractories, will be given. Pertinent examples in different systems will be discussed.

This paper compiles and reviews the last plenary lecture given by Professor Salvador De Aza on the subject.

Keywords: Phase equilibrium diagrams, refractories and $\mathrm{SiO}_{2}-\mathrm{CaO}, \mathrm{SiO}_{2}-\mathrm{MgO}, \mathrm{SiO}_{2}-\mathrm{CaO}-\mathrm{Al}_{2} \mathrm{O}_{3^{\prime}} \mathrm{MgO}-\mathrm{CaO}-\mathrm{SiO}_{2}-\mathrm{ZrO}_{2}, \mathrm{Al}_{2} \mathrm{O}_{3}-\mathrm{MgO}_{-}$ $\mathrm{CaO}$ systems.

\section{INTRODUCCIÓN}

El jueves 28 de octubre de 2010 a las 9:45 de la mañana, durante la celebración del 50 congreso de la Sociedad Española de Cerámica y Vidrio, el Profesor Salvador De Aza impartió su última conferencia plenaria [1]. Unos meses más tarde, el 13 de abril de 2011 a las 6:15 horas, fallecería inesperadamente como consecuencia de una neumonía a los 77 años de edad. Salvador De Aza ha sido pionero tanto en el establecimiento experimental como en el empleo de los diagramas de equilibrio de fases de sistemas oxídicos para el estudio, diseño y comprensión del comportamiento de los materiales cerámicos en España. Así como en su uso para abordar los procesos de su fabricación. Durante toda su carrera científica, fundamentó el estudio de los materiales cerámicos y por ende de los materiales refractarios, en el estudio teórico y experimental de los diagramas de equilibrio de fases, y su aplicación al diseño de materiales con comportamiento controlado para diferentes aplicaciones. Este artículo pretende recoger "el espíritu" de aquella conferencia invitada, en la que 
el Profesor expuso su visión de los diagramas de equilibrio de fases como una herramienta fundamental para el diseño y uso de los materiales refractarios. En concreto, el presente trabajo recopila y revisa dos conferencias impartidas sobre el tema $[1,2]$.

Dentro del campo de los materiales refractarios, la industria refractaria proporciona productos y servicios fundamentales en todos aquellos procesos industriales en los cuales es necesario la aplicación de calor. El diccionario de la lengua española, en su vigésima segunda edición, editada por La Real Academia Española define el término refractario de la siguiente manera: "dicho de un material: que resiste la acción del fuego sin alterarse" [3]. Sin embargo, esta definición es vaga e imprecisa. Una definición mucho más adecuada sería la siguiente: "todo material capaz de soportar, a temperaturas elevadas, las condiciones del medio en que está inmerso, durante un periodo de tiempo económicamente rentable, sin deterioro excesivo de sus propiedades físico-químicas" [4]. Definición, esta última, propuesta y usada por el Profesor Salvador De Aza en los innumerables cursos impartidos sobre la materia [4]. Las mencionadas condiciones del medio en que están inmersos los materiales refractarios hacen que estén expuestos a diferentes niveles de esfuerzos mecánicos y tensiones generadas térmicamente, a corrosión y erosión (proveniente de su contacto con otros sólidos, líquidos y/o gases), a difusión de gases y a abrasión mecánica a diferentes temperaturas [5, 6, 7, 8, 9]. Por todo ello son materiales muy complejos.

La aplicación de los diagramas de equilibrio de fases al diseño de nuevos materiales refractarios así como su uso para comprender sus comportamientos en servicio a altas temperaturas, ha sido, a lo largo del tiempo, una materia de controversia. La cual ha sido sustentada fundamentalmente por el célebre dictamen que J. W. Mellor hizo en 1907 [10]. En el que se expone que: “La reacción, entre los distintos componentes de un cuerpo cerámico, durante la cocción, se paraliza antes de que el sistema alcance el estado de equilibrio. Por lo tanto, la química de la cerámica es, en gran medida, una química de reacciones incompletas" [10]. Desde entonces, y por muchos años, dicha frase ha sido considerada como una ley fundamental por una gran número de ceramistas y refractaritas.

Tradicionalmente los materiales refractarios se han diseñado para que su composición esté próxima al equilibrio termodinámico, con la intención de que en servicio los cambios estén restringidos. Por otro lado, las altas temperaturas de uso de los materiales refractarios implican que estos materiales tiendan al equilibrio y que este se alcance durante su vida útil en servicio, al menos equilibrios locales. Adicionalmente, hoy en día, debido a los cambios habidos en los procesos tecnológicos y a la utilización de sofisticadas rutas de procesamiento, la cinética de reacción, entre los diferentes componentes de un material refractario, es suficientemente rápida como para alcanzar el equilibrio o quasi-equilibrio durante la cocción del material, así como posteriormente en servicio.

Así pues, en la actualidad, los diagramas de equilibrio son válidos no sólo para indicar la tendencia termodinámica de equilibrio durante la cocción del material refractario, sino también para predecir el estado final de equilibrio y, con una buena comprensión de las relaciones de equilibrio, también es posible conocer el camino a través del cual el sistema evoluciona hacia el estado final durante su aplicación [11, $12,13,14,15,16,17]$. Siendo especialmente útil este último punto en aquellos diseños, menos tradicionales, en los que se emplea la adición de materias primas que reaccionan de una manera controlada, durante el uso del material, para dar un determinado efecto favorable en las condiciones de trabajo a alta temperatura $[18,19,20,21]$.

En consecuencia, los diagramas de equilibrio son una potente herramienta para el diseño de nuevos materiales refractarios, así como para una mejor comprensión de su comportamiento en servicio en condiciones de alta temperatura y frente a agentes corrosivos [11-17, 22].

En el presente trabajo se pretende mostrar, utilizando algunos ejemplos representativos, cómo se puede explicar la fabricación de algunos materiales refractarios tradicionales y también cómo es posible mejorar y/o diseñar nuevos materiales refractarios mediante un uso adecuado de los diagramas de equilibrio de fases recogiendo "el espíritu" de aquella conferencia invitada [1].

\section{REFRACTARIOS DE SÍLICE}

Este apartado pretende poner de manifiesto cómo se puede explicar la fabricación de un material muy tradicional, los refractarios de sílice, y también cómo es posible mejorar su comportamiento mediante un control adecuado de las materias primas. Para ello, se utilizará sucesivamente la información suministrada por los sistemas binarios $\mathrm{SiO}_{2}-$ $\mathrm{CaO}[23,24]$ y $\mathrm{SiO}_{2}-\mathrm{MgO}[25,24]$ y del sistema ternario $\mathrm{SiO}_{2}$ $-\mathrm{CaO}-\mathrm{Al}_{2} \mathrm{O}_{3}[26,24]$.

La sílice pura es un compuesto refractario ya que su punto de fusión, a presión parcial de oxígeno de 0,21 atm., está a $1.723{ }^{\circ} \mathrm{C}$, según se indica en el sistema binario $\mathrm{SiO}_{2}-\mathrm{CaO}$ recogido en la Figura 1. Este compuesto tiene un gran número de fases polimórficas [27, 28], pero desde el punto de vista de sus aplicaciones como refractario las más significativas

TABLA 1. RANGOS DE ESTABILIDAD TERMODINÁMICA DE LAS FASES POLIMÓRFICAS MÁS SIGNIFICATIVAS DE LA SÍLICE Y DE LAS FORMAS DE ALTA Y BAJA TEMPERATURA DE CADA UNA.

\begin{tabular}{|c|c|c|c|c|}
\hline Fase polimórfica & Densidad $\left(\mathrm{g} \cdot \mathrm{cm}^{-3}\right)$ & Rango de estabilidad $\left({ }^{\circ} \mathrm{C}\right)$ & Temperatura de inversión $\left({ }^{\circ} \mathrm{C}\right)$ & $\mathrm{V}(\%)^{*}$ \\
\hline Cuarzo & 2,66 & Temperatura Ambiente -870 & $\alpha \leftrightarrow 573 \leftrightarrow \beta$ & $+0,8$ \\
\hline Tridimita & 2,30 & $870-1470$ & $\alpha \leftrightarrow 117 \leftrightarrow \alpha^{\prime} \leftrightarrow 163 \leftrightarrow \beta$ & $+0,2$ \\
\hline Cristobalita & 2,33 & $1470-1723$ & $\alpha \leftrightarrow 200-275 \leftrightarrow \beta$ & $+2,8$ \\
\hline
\end{tabular}

* Consultar Figura 2 donde se refleja claramente que en el caso de la tridimita el cambio de volumen es más paulatino y por tanto menos brusco. 
son: el cuarzo, la tridimita y la cristobalita [29]. La estructura cristalina de cada una de ellas está formada por tetraedros de $\mathrm{SiO}_{4}$ ordenados de distinta manera y cada polimorfo a su vez presenta dos o más tipos estructurales, con una simetría mayor según aumenta su temperatura de estabilidad $[27,28]$. En la Tabla 1 se muestran los rangos de estabilidad termodinámica de las fases polimórficas más significativas de la sílice y las formas de alta y baja temperatura de cada polimorfo [29].

El paso de una fase polimórfica a otra, por ejemplo de cuarzo a la tridimita, implica una trasformación de fase de tipo reconstructiva, es decir que la reordenación de los tetraedros de $\mathrm{SiO}_{4}$ se produce mediante una rotura de enlaces Si-O. Esto hace que se trate de un proceso muy lento cinéticamente. Además de tener lugar con un importante cambio de volumen. Ver Figura 2 [4, 29, 30]. Estos cambios necesitan del aporte de una gran cantidad de energía lo que hace que las fases sean metastables durante mucho tiempo, en el caso de los procesos de enfriamiento, y por tanto se pueden considerar irreversibles [27, 28, 29]. Así, no se ha detectado la tendencia a transformarse de nuevo en cuarzo, al enfriarse a temperaturas inferiores a $870{ }^{\circ} \mathrm{C}$, ni en la cristobalita ni en la tridimita [4].

Por otro lado, las transiciones entre las formas de alta y baja temperatura de cada polimorfo, por ejemplo de $(\alpha)$ cuarzo a $(\beta)$ cuarzo, son de tipo desplazativo, lo cual no implica la rotura de enlaces sólo un cambio en las dimensiones de la red. Estas transformaciones tienen lugar rápidamente y son reversibles a una temperatura de inversión constante y bien definida, ver Figura 2, y pueden ser repetidas una y otra vez sin la desintegración física de los cristales. Sin embargo, un ciclado térmico entre estas temperaturas de inversión puede deteriorar las propiedades físicas del refractario de sílice, debido a los cambios de volumen, y tener consecuencias catastróficas en el material.

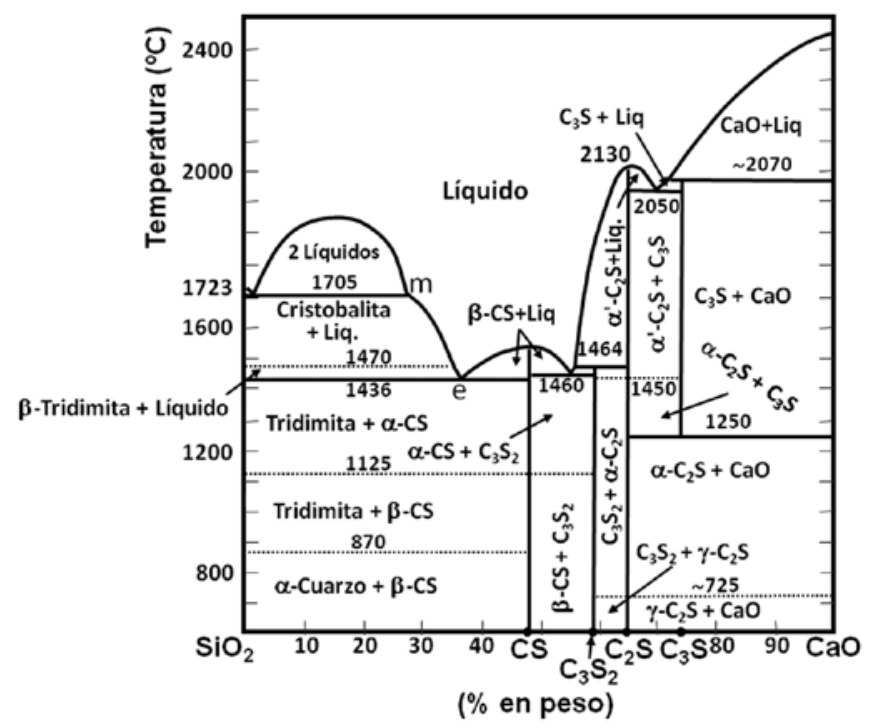

Figura 1. Sistema $\mathrm{SiO}_{2}-\mathrm{CaO}[23,24]$. Figura redibujada por S. De Aza [4]. En la presente figura para mayor claridad en la representación gráfica se ha empleado la nomenclatura empleada en cementos: $\mathrm{C}$ es $\mathrm{CaO}$, $\mathrm{S}$ es $\mathrm{SiO}_{2}$ (Ej. CS es $\mathrm{CaO} \cdot \mathrm{SiO}_{2}$ ó $\mathrm{CaSiO}_{3}$ ). Esta nomenclatura se usará en adelante en la figuras del trabajo.
Así pues, cuando un producto de sílice se trata térmicamente tienen lugar las transformaciones polimórficas necesarias para que se forme la fase de sílice estable a esa temperatura, la transformación se completará si el tiempo de permanencia a esa temperatura es suficiente para que incluso las trasformaciones más lentas tengan lugar. En los refractarios comerciales de sílice coexisten habitualmente cristobalita, timidita y algo de cuarzo residual, y según la aplicación del refractario pueden variar las proporciones de estos polimorfos [4].

Ahora bien, como es bien sabido por los refractaristas, los refractarios de sílice se suelen aglomerar con, aproximadamente, un $2 \%$ en peso de una lechada de cal, $\mathrm{CaO}$. Las cuestiones que surgen son: ¿porqué? y ¿porqué no utilizar otros aditivos como aglomerantes, por ejemplo óxido de magnesio, $\mathrm{MgO}$ ? Para contestar a estas preguntas hay que considerar y comprender, en primer lugar, la información contenida en el sistema $\mathrm{SiO}_{2}-\mathrm{CaO}[23,24]$, recogido en la Figura 1.

En dicho sistema, como se puede observar, la sílice, $\mathrm{SiO}_{2^{\prime}}$ es termodinámicamente compatible con el silicato cálcico o wollastonita, $\mathrm{CaSiO}_{3}\left(\mathrm{CaO} \cdot \mathrm{SiO}_{2}\right.$ ó CS), con el cual forma un subsistema binario con un punto eutéctico localizado a la temperatura de $1.436^{\circ} \mathrm{C}$ (designado " $\mathrm{e}$ ", en la Figura 1), presentando una cúpula de inmiscibilidad líquida, donde coexisten dos líquidos a $1.705^{\circ} \mathrm{C}$. Esto es, $18^{\circ} \mathrm{C}$ por debajo del punto de fusión de la sílice pura, que es $1.723^{\circ} \mathrm{C}$ según este diagrama.

Para poner de manifiesto claramente porqué la cal es añadida como aglomerante de la sílice, se muestra en la Figura 3, una ampliación del subsistema $\mathrm{SiO}_{2}-\mathrm{CaSiO}_{3}$.

Una vez aglomerados con un $2 \%$ en peso de $\mathrm{CaO}, \mathrm{los}$ ladrillos de sílice deben ser cocidos a $\mathrm{T} \geq 1.450^{\circ} \mathrm{C}$. Durante el tratamiento térmico (a temperaturas inferiores a $1.436{ }^{\circ} \mathrm{C}$,

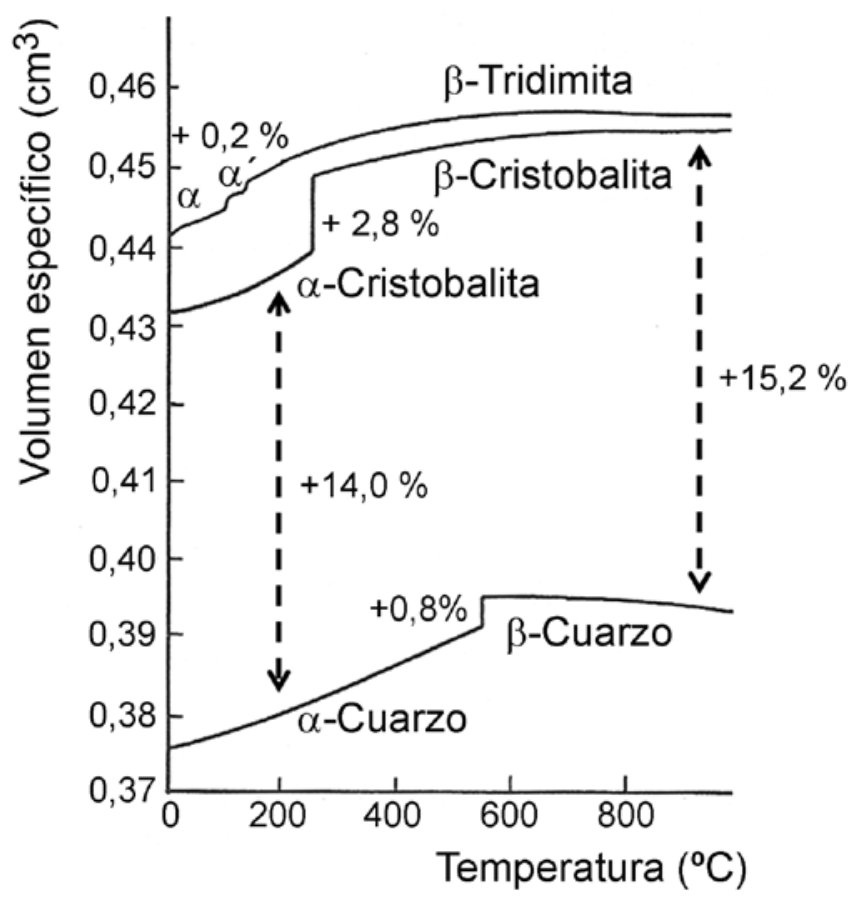

Figura 2. Dilatación térmica en función de las distintas fases del $\mathrm{SiO}_{2}$ presentes en el material $[4,7,8,9,29]$. 
consultar Figura 1 y 3) la cal tiende a reaccionar en estado sólido con la sílice para formar distintos silicatos cálcicos y al alcanzar una temperatura superior a $1.436{ }^{\circ} \mathrm{C}$ se forma una pequeña cantidad de fase líquida, ver Figura 3. Dicha fase líquida es una herramienta usada para facilitar que la fase polimórfica de baja temperatura de la sílice, el cuarzo (presente en la materia prima de partida) se transforme en el polimorfo tridimita. Fase estable a la temperatura del tratamiento térmico, coexistiendo con el mencionado líquido, ver Figura 3. Habitualmente, la presencia de pequeñas cantidades de impurezas hace que las temperaturas de trasformación sean ligeramente inferiores a las indicadas en el diagrama de la Figura 3 para compuestos puros. Por tanto, la fase que se forma durante los primeros estadios del tratamiento térmico a partir del cuarzo es la cristobalita, que posteriormente con el tiempo y la temperatura se transforma en tridimita. La tridimita es la fase polimórfica que interesa tener en el refractario final, ya que, aparte de ser la fase estable a las temperaturas de trabajo de estos materiales, es el polimorfo que presenta menores cambios de volumen $(0,2 \%)$ asociado a las transformaciones entre las formas de baja y alta temperatura, $(\alpha) \longleftrightarrow(\beta)$, siendo además un cambio menos brusco $[4,7,8,9,29]$. Consultar Figura 2. Se minimiza así el riesgo de problemas por cambios bruscos de volumen del material durante los ciclos de enfriamiento-calentamiento en servicio. Lo cual evita la generación de grandes tensiones térmicas durante los ciclos térmicos que puedan deteriorar el material. Además, la proporción de fase líquida generada, calculada mediante el uso de la regla de la palanca [31, 32], es de tan solo un 5,5\% en peso y su viscosidad es relativamente alta, debido a su alto contenido de sílice que es $\sim 65 \%$ en peso (ver Figura 3), por lo que su presencia no es un problema para el material.

Si estos ladrillos de sílice son llevados en servicio hasta $1.700^{\circ} \mathrm{C}$, solo $23^{\circ} \mathrm{C}$ por debajo del punto de fusión de la sílice pura $\left(1.723^{\circ} \mathrm{C}\right)$, el contenido de líquido, a dicha temperatura, es del $7.1 \%$ en peso. Esto representa un incremento, en el contenido de líquido, de tan solo un $1.6 \%$ en peso, con

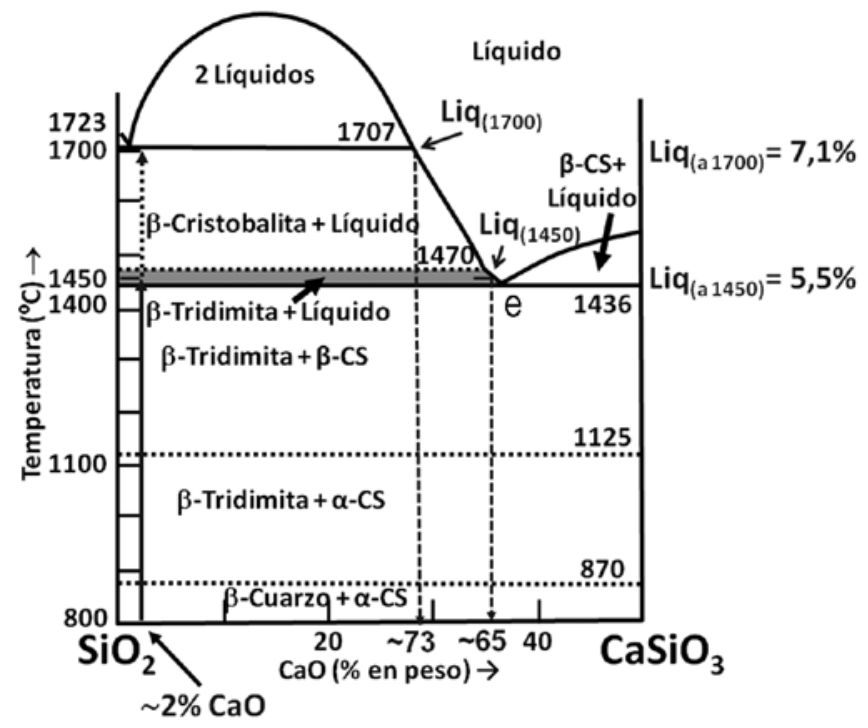

Figura 3. Subsistema $\mathrm{SiO}_{2}-\mathrm{CaSiO}_{3}[23,24]$. Figura redibujada por S. De Aza [4]. respecto al de la temperatura de cocción a $1.450^{\circ} \mathrm{C}$. Siendo ahora además su viscosidad más alta, que en el caso previo, ya que el contenido en sílice en el líquido ha aumentado, ahora es del orden del $73 \%$ en peso (ver Figura 3).

Al mismo tiempo, todo lo expuesto hace que la microestructura, generada en estos ladrillos, esté formada por una matriz constituida fundamentalmente por pequeños cristales de tridimita con algunos granos residuales de cristobalita y de cuarzo (parcialmente transformados en cristobalita). Los cristales de cristobalita se forman a partir de los bordes de los granos originales de cuarzo dando una morfología muy característica (con cierta similitud a las escamas de pescado) ver Figura 4. Entre los granos de tridimita se encuentra la pequeña proporción de fase vítrea, localizada en áreas totalmente aisladas de morfología triangular (puntos triples). Este tipo de microestructura justifica las excelentes propiedades mecánicas a alta temperatura de los ladrillos de sílice así fabricados [33].

Por lo tanto, estos ladrillos deben calcinarse a las temperaturas apropiadas y durante los tiempos necesarios para que la transformación de la materia prima, cuarcita, a tridimita o cristobalita y tridimita sea completa con objeto de minimizar el riesgo de expansiones posteriores en servicio. Como se ha expuesto, hay que realizar una cocción muy cuidadosa que comienza por una calcinación a temperaturas del orden de $1.450{ }^{\circ} \mathrm{C}$ por largos períodos de tiempo. La velocidad de enfriamiento debe controlarse cuidadosamente con objeto de acomodar las inversiones de la las fases presentes (ver Figura 2) ya que implican cambios de volumen particularmente rápidos en algunos de los casos.

Todo lo anterior justifica el porqué la cal es utilizada como aglomerante de la sílice. Ahora bien, llegados a este punto, puede surgir la pregunta: ¿porqué no utilizar otros aditivos como aglomerantes?, por ejemplo, el óxido de magnesio $(\mathrm{MgO})$.

Para encontrar la respuesta hay que recurrir al sistema $\mathrm{SiO}_{2}$ - $\mathrm{MgO}[25,24]$. En este sistema, la sílice es termodinámicamente

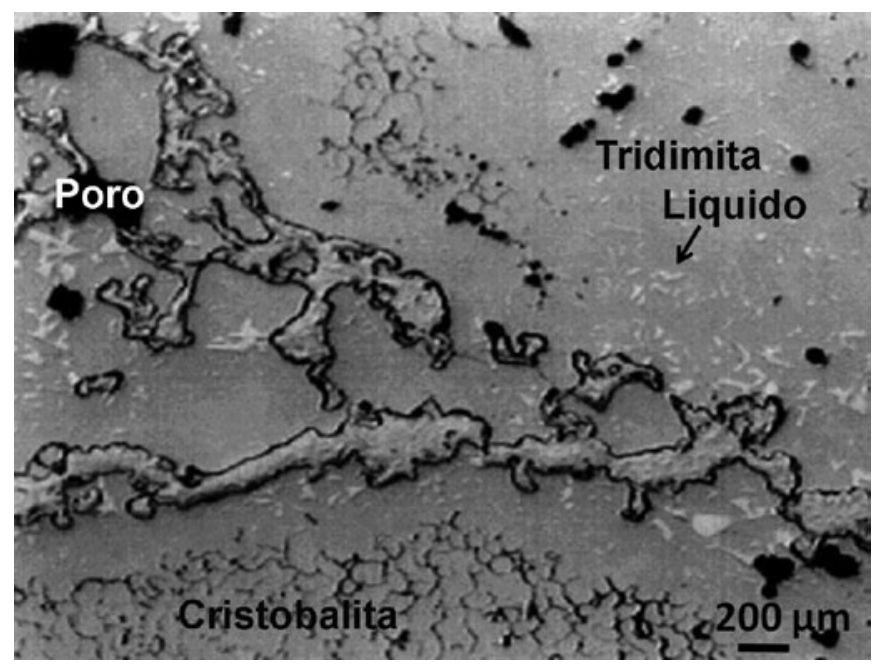

Figura 4. Imagen de Microscopía Óptica de Luz Reflejada (MOLR) de una superficie pulida de una muestra de un refractario de sílice de la bóveda de un horno, tipo balsa, de vidrio. Composición (en \% en peso): $96 \%$ de $\mathrm{SiO}_{2}, 0,60 \% \mathrm{Al}_{2} \mathrm{O}_{3^{\prime}} 0,05 \% \mathrm{Na}_{2} \mathrm{O}$, otros 3,35\%. Densidad aparente $1,78 \mathrm{~g} \cdot \mathrm{cm}^{-3}$. Porosidad abierta $22 \%$. Resistencia compresión en frío $30 \mathrm{MPa}$. 
compatible con el silicato de magnesio o enstatita, $\mathrm{MgSiO}_{3^{\prime}}$ formando un subsistema binario, el cual se presenta en la Figura 5. Como se puede apreciar, se trata, a primera vista, de un subsistema similar al $\mathrm{SiO}_{2}-\mathrm{CaSiO}_{3}$ expuesto anteriormente en la Figura 3. En el presente caso el subsistema presenta un punto eutéctico localizado a $1.543^{\circ} \mathrm{C}$, más alto que en el caso anterior, presentando igualmente un gran campo de inmiscibilidad líquida donde coexisten dos líquidos, a partir de $1.703^{\circ} \mathrm{C}$.

Sin embargo, como se puede apreciar, el campo de estabilidad de la tridimita, que por las razones expuestas anteriormente es la fase polimórfica de la sílice que interesa obtener en el material, se extiende hasta la temperatura de $1.470^{\circ} \mathrm{C}$. Estando esta por debajo de la primera formación de fase líquida, la cual tiene lugar a $1.543^{\circ} \mathrm{C}$. Este hecho dificulta seriamente la transformación polimórfica del cuarzo $(\beta)$ a tridimita, que tendría que tener lugar en estado sólido, evitándola, y justificando que el $\mathrm{MgO}$ no pueda usarse como aglomerante de la sílice.

Otra cuestión relacionada con los ladrillos de sílice, es el pernicioso efecto de la presencia de pequeñas cantidades de alúmina sobre su refractariedad. ¿Cuál es la causa? Para explicar dicho efecto se va a considerar el sistema ternario $\mathrm{SiO}_{2}-\mathrm{CaO}-\mathrm{Al}_{2} \mathrm{O}_{3}[26,24]$, del cual se muestra solo la región rica en sílice en la Figura 6 .

Como se puede apreciar, la presencia de alúmina, $\mathrm{Al}_{2} \mathrm{O}_{3^{\prime}}$ reduce drásticamente el área de inmiscibilidad líquida dentro del sistema ternario. Supongamos, que se utiliza, como en el caso anterior, un $2 \%$ en peso de cal como aglomerante, y que la materia prima de sílice contiene un $1,0 \%$ en peso de $\mathrm{Al}_{2} \mathrm{O}_{3}$. La localización de la composición del material caerá fuera de la zona de inmiscibilidad y dentro del triángulo

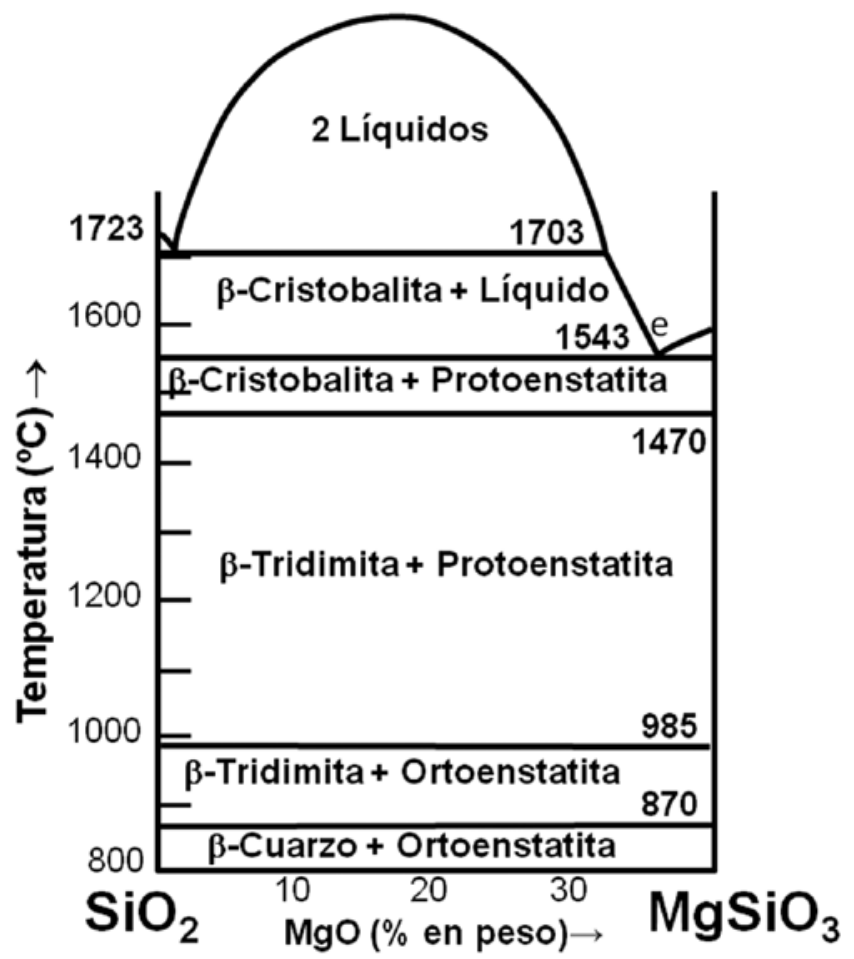

Figura 5. Subsistema $\mathrm{SiO}_{2}-\mathrm{MgSiO}_{3}[25,24]$ Figura redibujada por $\mathrm{S}$. De Aza [4]. de compatibilidad: sílice-anortita-wollastonita, cuyo punto eutéctico tiene lugar a $1.170^{\circ} \mathrm{C}$. Ver Figura 6. En consecuencia, durante la cocción, la primera formación de fase líquida tendrá lugar a dicha temperatura. A medida que esta se eleva la composición del líquido se moverá a lo largo de la línea eutéctica binaria que separa los campos primarios de cristalización de la sílice y la wollastonita, desapareciendo la anortita del material, en ese preciso instante, hasta alcanzar una temperatura donde la composición del líquido, la composición de partida del refractario y la sílice coinciden en línea recta. Ver línea dibujada en el diagrama de la Figura 6 a este efecto. Supongamos que cocemos el material a $1.450^{\circ} \mathrm{C}$, como en el primer caso expuesto, dentro de la zona de estabilidad de la tridimita. La composición del líquido se moverá a lo largo de dicha línea recta, desapareciendo en ese momento la wollastonita del material, hasta alcanzar la isoterma de $1.450^{\circ} \mathrm{C}$. A dicha temperatura el porcentaje de fase líquida coexistiendo con la tridimita, calculado mediante la regla de la palanca [31,32] será del 10\% peso. Lo cual representa duplicar prácticamente la cantidad de líquido formado en ausencia de la impureza de alúmina (comparar con la Figura 3). Si la temperatura en servicio se eleva hasta $1.600^{\circ} \mathrm{C}$ el porcentaje de líquido será del $17,5 \%$, lo que representa un incremento del $146 \%$ sobre el contenido de líquido calculado previamente a $1.700^{\circ} \mathrm{C}$ en ausencia de $\mathrm{Al}_{2} \mathrm{O}_{3}$ (comparar nuevamente con la Figura 3). Se puede ver así, que se produce un incremento muy sustancial de la fase líquida, lo que justifica el efecto tan perjudicial de las pequeñas cantidades de alúmina presentes en la materia prima de sílice.

Considérese ahora que el contenido de $\mathrm{Al}_{2} \mathrm{O}_{3}$ en la materia prima de sílice es del 0,5\% en peso y que se utiliza el mismo porcentaje de cal como aglomerante ( $2 \%$ en peso). En este

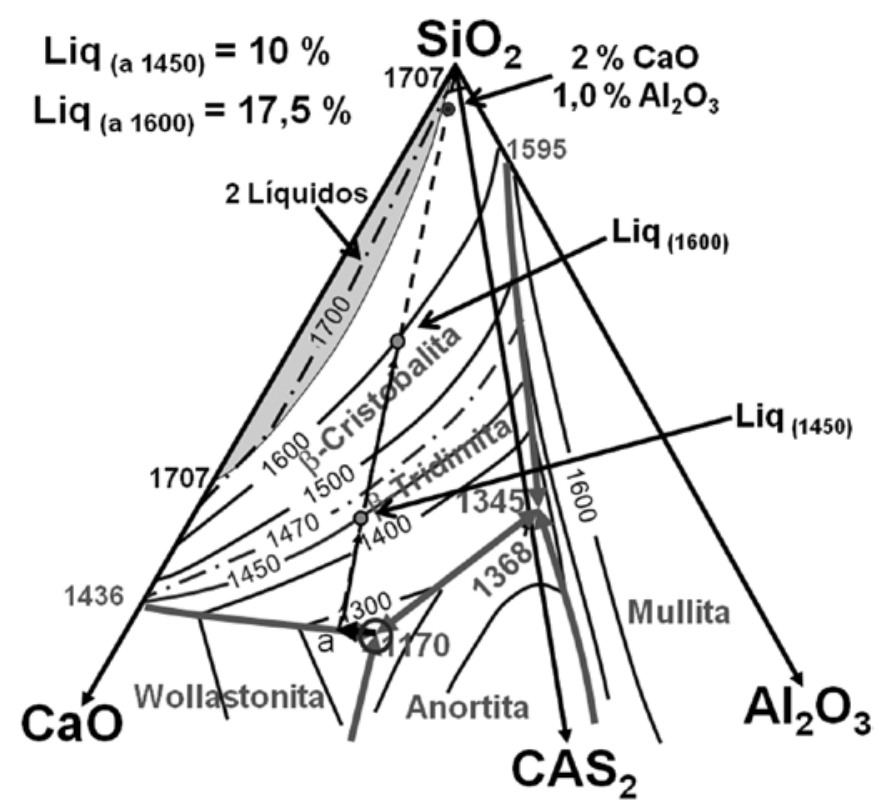

Figura 6. Región de alto contenido en $\mathrm{SiO}_{2}$ del sistema ternario $\mathrm{SiO}_{2}$ - $\mathrm{CaO}-\mathrm{Al}_{2} \mathrm{O}_{3}[26,24]$. Región ampliada y dibujada por S. De Aza [4]. La figura ilustra el efecto de la presencia de una impureza del 1,0\% de alúmina sobre la refractariedad de un ladrillo de sílice aglomerado con un $2 \%$ de $\mathrm{CaO}$. 
caso, la composición del material estará localizada dentro del área de inmiscibilidad sobre la isoterma de $1700^{\circ} \mathrm{C}$. Ver Figura 7. Durante la cocción, la primera aparición de líquido tendrá lugar a $1.170^{\circ} \mathrm{C}$, como en el caso anterior. Igualmente, a medida que la temperatura se incrementa, la composición del líquido migrará a lo largo de la línea eutéctica binaria que separa los campos primarios de cristalización de la sílice y la wollastonita, hasta llegar a una temperatura donde la composición del líquido estará en línea recta con la composición de partida y la sílice. A partir de ahí, como ya se ha visto, si la temperatura aumenta, la composición del líquido se irá moviendo a lo largo de dicha línea recta, desapareciendo del material la wollastonita. Así por ejemplo, a la temperatura de cocción del material de sílice, $1.450^{\circ} \mathrm{C}$, el porcentaje de fase líquida, calculado por la regla de la palanca [31, 32], será tan solo del $6,4 \%$ en peso, es decir un incremento con respecto al caso de la ausencia de $\mathrm{Al}_{2} \mathrm{O}_{3}$ de tan solo un $0,9 \%$ (ver Figura 3). Si la temperatura en servicio se incrementa hasta $1.600^{\circ} \mathrm{C}$, el porcentaje se líquido se incrementará hasta el 7,9\%, es decir, un $0,8 \%$ más que en el caso de la ausencia de $\mathrm{Al}_{2} \mathrm{O}_{3}$ a $1.700^{\circ} \mathrm{C}$ (ver Figura 3). En ambos casos, el contenido de líquido es similar a los valores en ausencia de la impureza de alúmina, si bien en el segundo caso la temperatura es $100^{\circ} \mathrm{C}$ inferior a la considerada previamente, puesto que si se alcanzasen los $1700^{\circ} \mathrm{C}$ el material de sílice fundiría completamente en este último caso (ver Figura 7).

Todo lo expuesto justifica porqué el contenido de alúmina en los ladrillos de sílice debe restringirse, para aplicaciones superiores a $1600^{\circ} \mathrm{C}$, a valores por debajo del $0,5 \%$ en peso.

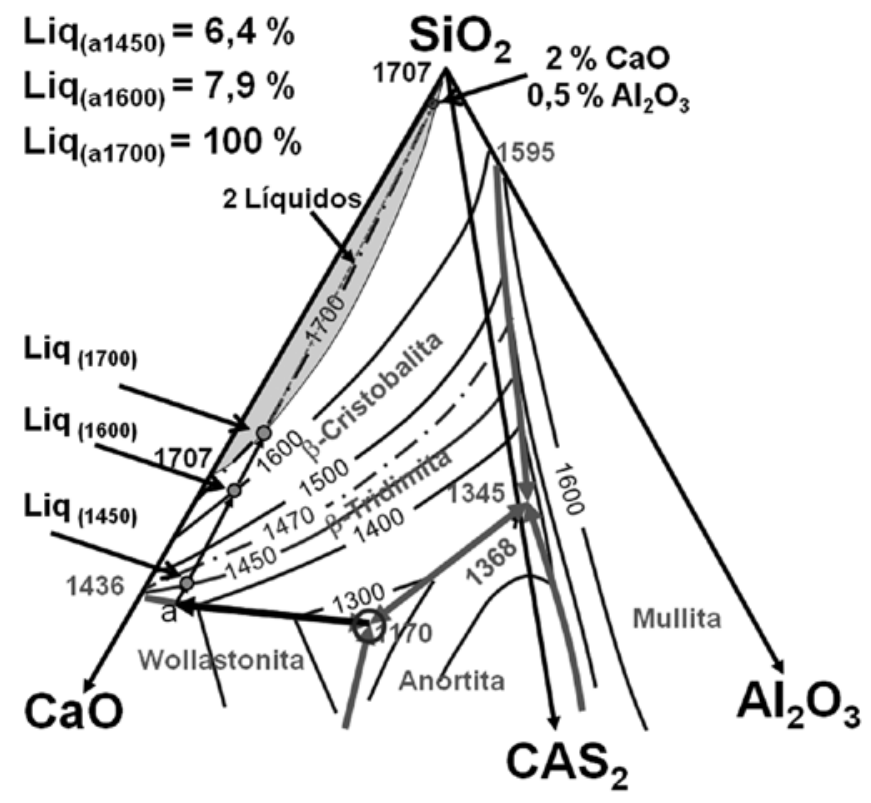

Figura 7. Región de alto contenido en $\mathrm{SiO}_{2}$ del sistema ternario $\mathrm{SiO}_{2}$ - $\mathrm{CaO}-\mathrm{Al}_{2} \mathrm{O}_{3}[26,24]$. Región ampliada y dibujada por S. De Aza [4]. La figura ilustra el efecto de la presencia de una impureza del 0,5\% de alúmina sobre la refractariedad de un ladrillo de sílice aglomerado con un $2 \%$ de $\mathrm{CaO}$.

\section{REFRACTARIOS DE MAGNESIA}

Una vez expuesto como los diagramas de equilibrio de fases pueden explicar adecuadamente el proceso de fabricación de los refractarios de sílice, así como el pernicioso efecto de la presencia de pequeñas cantidades de alúmina como impurezas, se describirá a continuación otro ejemplo, en este caso de diseño de nuevos materiales refractarios, en relación con refractarios de magnesia, haciendo uso para ello del sistema $\mathrm{MgO}-\mathrm{CaO}-\mathrm{SiO}_{2}-\mathrm{ZrO}_{2}[34,24]$.

El objetivo original del estudio de este sistema ternario fue examinar el posible desarrollo de nuevos refractarios de magnesia, $\mathrm{MgO}$, conteniendo circonato cálcico, $\mathrm{CaZrO}_{3}$ ( $\mathrm{CaO} \cdot \mathrm{ZrO}_{2}$ ó CZ), o circona, $\mathrm{ZrO}_{2}$, como segundas fases [19, $34,35,36,37,38,39]$. Substituyendo de esta manera el papel de la espinela de cromo en los materiales refractarios de magnesia-cromo y evitando así el uso de cromo [40, 41]. Estos materiales son idóneos para su utilización como refractarios que se empleen en la zona de clinquerización de los hornos de cemento Portland y en las cucharas de colada [19, 34, 35, 36, 37,39].

Para investigar si se daban los requerimientos previos necesarios dentro de este sistema ternario, se empezó por determinar las compatibilidades en estado sólido de la magnesia en dicho sistema [34, 24], las cuales se muestran en la Figura 8. En dicha figura, en la leyenda adjunta, se indican las distintas compatibilidades establecidas.

Como se puede apreciar en la figura, existen seis tetraedros de compatibilidad conteniendo $\mathrm{MgO}$. Tres de ellos donde el $\mathrm{CaZrO}_{3}$ coexiste con el $\mathrm{MgO}$ (compatibilidades etiquetadas como 1,2 y 3 en la leyenda de la Figura 8); dos donde el $\mathrm{ZrO}_{2}$ coexiste con el $\mathrm{MgO}(5$ y 6) y uno donde ambos coexisten con el $\mathrm{MgO}(4)$.

Una vez confirmadas las compatibilidades existentes, los requisitos que debe cumplir una segunda fase sólida que coexista con el $\mathrm{MgO}$ en estos refractarios han de ser:

$1^{\circ}$. Deberá ser una fase altamente refractaria.

$2^{\circ}$. No debe formar puntos de fusión bajos con el $\mathrm{MgO}$ ni con los demás componentes de los refractarios de magnesia, y

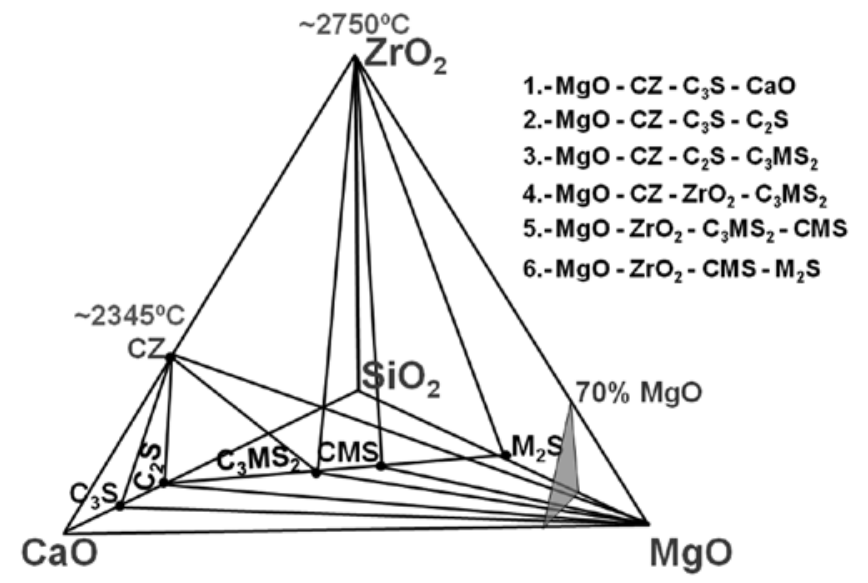

Figura 8. Compatibilidades en estado sólido del $\mathrm{MgO}$ dentro del sistema cuaternario $\mathrm{MgO}-\mathrm{CaO}-\mathrm{SiO}_{2}-\mathrm{ZrO}_{2}$ según S. De Aza, C. Richmond y J. White [34, 24]. En la leyenda adjunta, se indican las compatibilidades establecidas. Adicionalmente se indica en la figura el plano correspondiente al $70 \%$ en peso de $\mathrm{MgO}$. 
$3^{\circ}$. Debe mantener un alto grado de contacto directo, sólidosólido, con el $\mathrm{MgO}$, incluso en presencia de una fase líquida a altas temperaturas.

Tanto el $\mathrm{CaZrO}_{3}$ como el $\mathrm{ZrO}_{2}$ satisfacen el primer requerimiento, con puntos de fusión superiores a $2.000^{\circ} \mathrm{C}$, como se muestra en la Figura 8 donde se indican sus respectivos puntos de fusión.

Para evaluar el siguiente requerimiento $\left(\mathrm{el} 2^{\circ}\right)$, se procedió a establecer la proyección del volumen primario de cristalización del $\mathrm{MgO}$, para el plano del $70 \%$ en peso de $\mathrm{MgO}$, la cual se muestra en la siguiente Figura 9 [34, 24]. En dicha figura, en la leyenda adjunta, se indican las distintas compatibilidades.

Como puede apreciarse, las temperaturas más elevadas de primera formación de fase liquida se presentan en las compatibilidades de fases indicadas como 1 y 2 en la Figura 9, donde el $\mathrm{CaZrO}_{3}$ coexiste con $\mathrm{MgO}, \mathrm{CaO}$ y $\mathrm{Ca}_{3} \mathrm{SiO}_{5}\left(3 \mathrm{CaO} \cdot \mathrm{SiO}_{2}\right.$ ó C $\left.\mathrm{C}_{3} \mathrm{~S}\right)$ y con $\mathrm{MgO}, \mathrm{Ca}_{3} \mathrm{SiO}_{5}$ y $\mathrm{Ca}_{2} \mathrm{SiO}_{4}\left(2 \mathrm{CaO} \cdot \mathrm{SiO}_{2}\right.$ ó $\left.\mathrm{C}_{2} \mathrm{~S}\right)$ respectivamente. $\mathrm{El}$ resto de las compatibilidades de fases presentan, como se puede apreciar, puntos relativamente bajos de fusión.

En consecuencia, el $\mathrm{CaZrO}_{3}$ cumple el $2^{\circ}$ requisito previamente expuesto, mientras que la $\mathrm{ZrO}_{2}$ no.

Si se observa con detenimiento la proyección recogida en la Figura 9 se puede apreciar que la línea que une el $\mathrm{CaZrO}_{3}$ con el $\mathrm{Ca}_{2} \mathrm{SiO}_{4^{\prime}}$ es en realidad la proyección de la sección $\mathrm{CaZrO}_{3}-$ $\mathrm{Ca}_{2} \mathrm{SiO}_{4}-\mathrm{MgO}$, la cual es un sistema pseudoternario, cuyo punto invariante peritéctico, indicado como " $b$ " en la Figura 9, tiene lugar a $1.710^{\circ} \mathrm{C}$. Dicho sistema se muestra en la Figura 10. Para diseñar y poder formular los materiales de $\mathrm{MgO}$ contenido $\mathrm{CaZrO}_{3}$ como segunda fase sólida este es el sistema que se debe utilizar.

Para una composición conteniendo, por ejemplo, el $80 \%$ en peso de $\mathrm{MgO}$, como se puede deducir haciendo uso del sistema recogido en la citada Figura 10, el $\mathrm{CaZrO}_{3}$ permanecerá como segunda fase sólida estable a alta temperatura, junto con el $\mathrm{MgO}$ y una fase líquida. Esto será así hasta una temperatura máxima que dependerá de la relación $\mathrm{CaZrO}_{3} /$

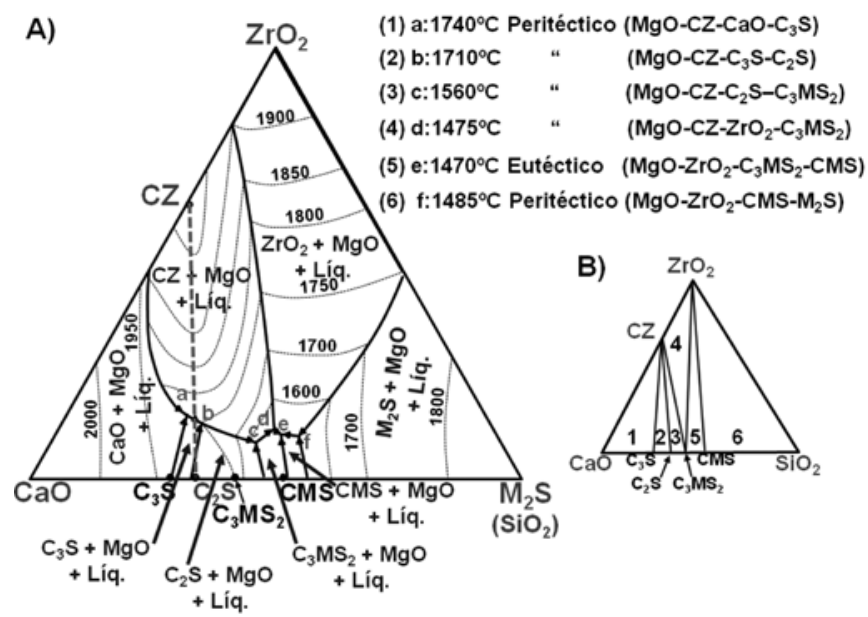

Figura 9. A) Proyección del volumen primario de cristalización del $\mathrm{MgO}$, para el plano del $70 \%$ en peso de $\mathrm{MgO}$ (consultar también la Figura 8). B) Proyección desde el vértice del $\mathrm{MgO}$ de los tetraedros de compatibilidad en estado sólido. En la leyenda adjunta, se indican las distintas compatibilidades. Según S. De Aza, C. Richmond y J. White $[34,24]$. Adicionalmente se indica la línea que une el $\mathrm{CaZrO}_{3}$ con el $\mathrm{Ca}_{2} \mathrm{SiO}_{4}$ (línea CZ-C 2 en el dibujo).
$\mathrm{Ca}_{2} \mathrm{SiO}_{4}$ existente en la formulación de la matriz del refractario diseñado.

Así, por ejemplo, para una relación en peso de $\mathrm{CaZrO}_{3} /$ $\mathrm{Ca}_{2} \mathrm{SiO}_{4}$ igual a 0,91, lo que conlleva una formulación de la matriz de partida de: $80 \% \mathrm{MgO}, 9,53 \% \mathrm{CaZrO}_{3}$ y 10,47\% $\mathrm{Ca}_{2} \mathrm{SiO}_{4}$, representada en la Figura 10 como "A", el $\mathrm{CaZrO}_{3}$ permanece como segunda fase sólida estable hasta $1.900^{\circ} \mathrm{C}$. Ver línea discontinua dibujada en la Figura 10 a tal efecto.

Para otra relación, por ejemplo, $\mathrm{CaZrO}_{3} / \mathrm{Ca}_{2} \mathrm{SiO}_{4}$ igual a 3,27 , la composición de la matriz de partida del material es ahora: $80 \% \mathrm{MgO}, 15,32 \% \mathrm{CaZrO}_{3}$ y $4,68 \% \mathrm{Ca}_{2} \mathrm{SiO}_{4^{\prime}}$ representada en la Figura 10 como " $\mathrm{B}$ ", la fase $\mathrm{CaZrO}_{3}$ permanece ahora como segunda fase sólida estable, junto con el $\mathrm{MgO}$, hasta incluso $\operatorname{los} 2.000^{\circ} \mathrm{C}$. Ver línea discontinua dibujada en la Figura 10 a tal efecto.

En ambos casos, el contenido de fase líquida es del orden del $22 \%$ en peso a las temperaturas indicadas.

Por otro lado el $\mathrm{CaZrO}_{3}$ presenta, como se puede observar en la Figura 11 A, un alto grado de contactos directos, solidosólido, con la periclasa, no solo en estado sólido, como se muestra en la microfotografía, donde coexisten $\mathrm{MgO}-\mathrm{CaZrO}_{3}-$ $\mathrm{Ca}_{2} \mathrm{SiO}_{4}$ a $1.700^{\circ} \mathrm{C}$, sino también a más altas temperaturas, como se muestra en la microfotografía recogida en la Figura 11 B, donde coexisten $\mathrm{MgO}-\mathrm{CaZrO}_{3}$-Líquido a $1.900^{\circ} \mathrm{C}$.

Por consiguiente, el $\mathrm{CaZrO}_{3}$ satisface completamente el $3^{\circ}$ requerimiento expuesto previamente.

Así pues, se pueden diseñar y fabricar materiales refractarios de $\mathrm{MgO}$, conteniendo $\mathrm{CaZrO}_{3}$ como segunda fase sólida estable, mediante el uso apropiado del sistema cuaternario $\mathrm{MgO}-\mathrm{CaO}-\mathrm{SiO}_{2}-\mathrm{ZrO}_{2}[34,24]$ o bien mediante el sistema pseudoternario $\mathrm{MgO}-\mathrm{CaZrO}_{3}-\mathrm{Ca}_{2} \mathrm{SiO}_{4}$ [34, 24], diseñando sus matrices con la adición de pequeñas adiciones de $\mathrm{ZrO}_{2}[37,39]$ o mediante adiciones controladas de dolomita, $\mathrm{MgCa}\left(\mathrm{CO}_{3}\right)_{2^{\prime}}$ mas circón, $\mathrm{ZrSiO}_{4^{\prime}}[19,35,36]$, siendo estos materiales idóneos para su utilización en la zona de clinquerización de los hornos de cemento Portland y en las cucharas de colada [34, 35, 37].

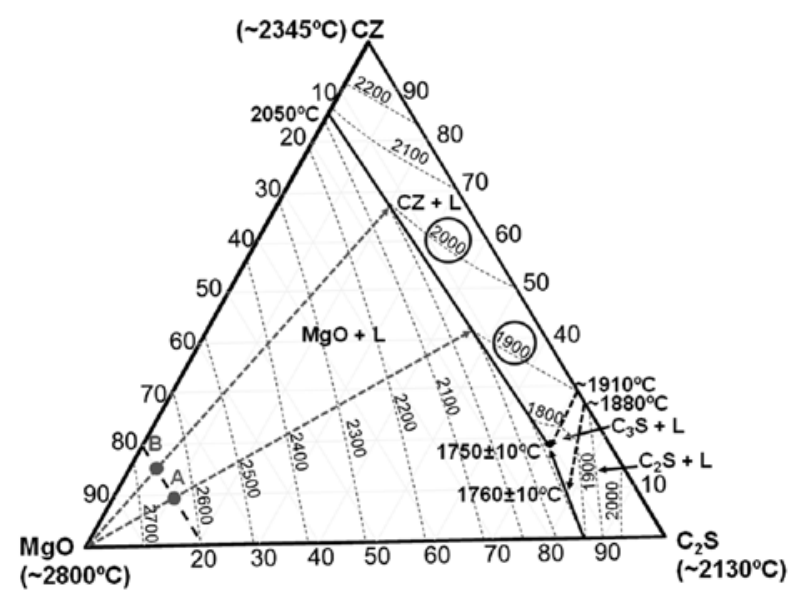

Figura 10. Sistema pseudoternario $\mathrm{CaZrO}_{3}-\mathrm{CaSiO}_{4}-\mathrm{MgO}$. La proyección de este sistema sobre la Figura 9 A es la línea $\mathrm{CaZrO}_{3}-\mathrm{Ca}_{2} \mathrm{SiO}_{4}$ indicada en dicha figura. Al tratarse de un sistema pseudoternario su punto invariante peritéctico se localiza fuera del plano $\mathrm{CaZrO}_{3}$ $\mathrm{Ca}_{2} \mathrm{SiO}_{4}-\mathrm{MgO}$. Dicho punto está indicado como " $\mathrm{b}$ " en la Figura $9 \mathrm{~A}$ $\left(1.710^{\circ} \mathrm{C}\right)[34,24]$. Formulaciones indicadas como A: $\mathrm{CaZrO}_{3} / \mathrm{Ca}_{2} \mathrm{SiO}_{4}$ $=0,91$ y B: $\mathrm{CaZrO}_{3} / \mathrm{Ca}_{2} \mathrm{SiO}_{4}=3,27$. 


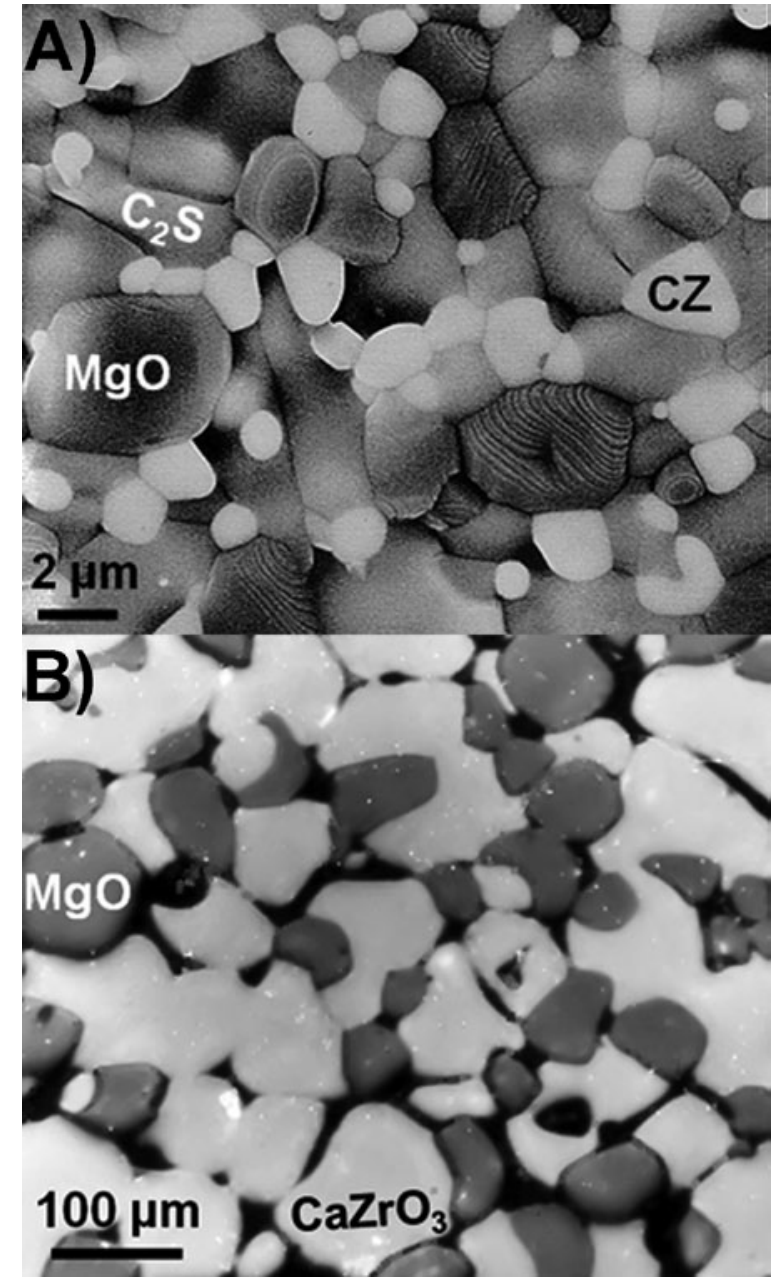

Figura 11 A) Imagen de Microscopía Electrónica de Barrido (MEB) de una superficie de fractura de un refractario de $\mathrm{MgO}-\mathrm{CaZrO}_{3}$ - $\mathrm{Ca}_{2} \mathrm{SiO}_{4}$ a $1700^{\circ} \mathrm{C}$ (estado sólido). Se indican las distintas fases presentes sobre la microestructura de la imagen [35]. B) Imagen de Microscopía Óptica de Luz Reflejada (MOLR) de una superficie pulida de una muestra de $\mathrm{MgO}, \mathrm{CaZrO}_{3}$ y Líquido a $1900^{\circ} \mathrm{C}$. Las áreas negras son poros y fase liquida atacada químicamente con objeto de revelar la microestructura [4].

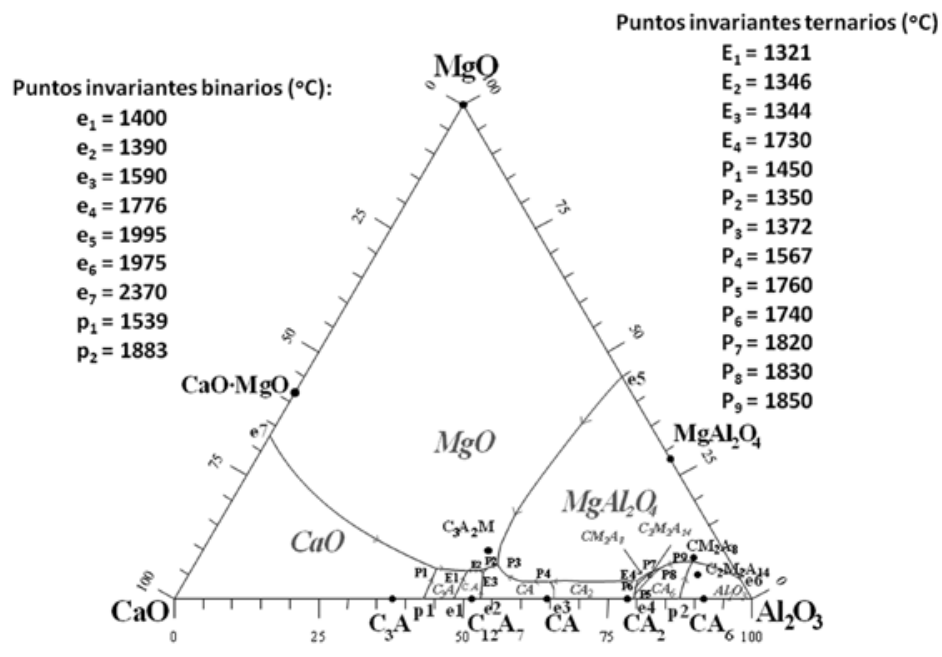

Figura 12. Sistema ternario $\mathrm{Al}_{2} \mathrm{O}_{3}-\mathrm{MgO}-\mathrm{CaO}$ según A.H. De Aza et al. $[45,46,44,24]$. Se muestran los campos primarios de cristalización de las diferentes fases, existentes dentro del sistema, así como los diversos puntos invariantes, tanto binarios como ternarios.

\section{REFRACTARIOS DE ALTA ALÚMINA CONTENIENDO ESPINELA}

El tercer ejemplo, que se va a presentar, hace referencia al reciente interés en el desarrollo tanto de ladrillos como de hormigones de alta alúmina conteniendo espinela $[42,43]$.

El diseño científico de estos materiales requiere el conocimiento previo de las relaciones de equilibrio de fases en el sistema $\mathrm{Al}_{2} \mathrm{O}_{3}-\mathrm{MgO}-\mathrm{CaO}$, especialmente en la región de altos contenidos de $\mathrm{Al}_{2} \mathrm{O}_{3}$. Los datos correspondientes a esta región del sistema no habían sido determinados previamente. En consecuencia, el sistema tuvo que ser estudiado [44, 45, 46, 47], previamente al desarrollo del material, y los resultados se exponen en la Figura 12.

La Figura 12 muestra los campos primarios de cristalización de las diferentes fases, existentes dentro del sistema, así como los diversos puntos invariantes, tanto binarios como ternarios.

Como se puede apreciar en la región de alto contenido en alúmina todos los puntos invariantes son más altos de $1.700^{\circ} \mathrm{C}$ e incluso algunos de ellos son superiores a $1.800^{\circ} \mathrm{C}$.

$\mathrm{Si}$ estos valores se comparan con las temperaturas de los puntos invariantes que rodean al campo primario de cristalización del $\mathrm{Al}_{2} \mathrm{O}_{3}$ en el sistema $\mathrm{Al}_{2} \mathrm{O}_{3}-\mathrm{SiO}_{2}-\mathrm{CaO}$ [26, 24] (Figura 13), donde tradicionalmente se fabrican dichos materiales, se puede apreciar la ventaja de utilizar la espinela $\left(\mathrm{MgAl}_{2} \mathrm{O}_{4}\right)$ o el hexaluminato cálcico $\left(\mathrm{CaAl}_{12} \mathrm{O}_{19}\right.$ ó $\mathrm{CaO} \cdot 6 \mathrm{Al}_{2} \mathrm{O}_{3}$ ó $\left.\mathrm{CA}_{6}\right)$ en vez de la mullita $\left(\mathrm{Al}_{6} \mathrm{Si}_{2} \mathrm{O}_{13}\right.$ ó $3 \mathrm{Al}_{2} \mathrm{O}_{3} \cdot 2 \mathrm{SiO}_{2}$ ó $\left.\mathrm{A}_{3} \mathrm{~S}_{2}\right)$ como segundas fases sólidas en los refractarios de alta alúmina, debido a que, en este caso, los puntos invariantes, como podemos ver, son considerablemente más bajos que los previamente mostrados.

Este hecho justifica sobradamente el interés por desarrollar tanto ladrillos como hormigones refractarios de alta alúmina conteniendo espinela.

Para explicar con mayor detalle cómo se pueden diseñar estos materiales se muestra una ampliación de la región de alta alúmina, en la Figura 14.

Las matrices, de estos materiales de alta alúmina conteniendo espinela, pueden ser diseñadas utilizando cementos de

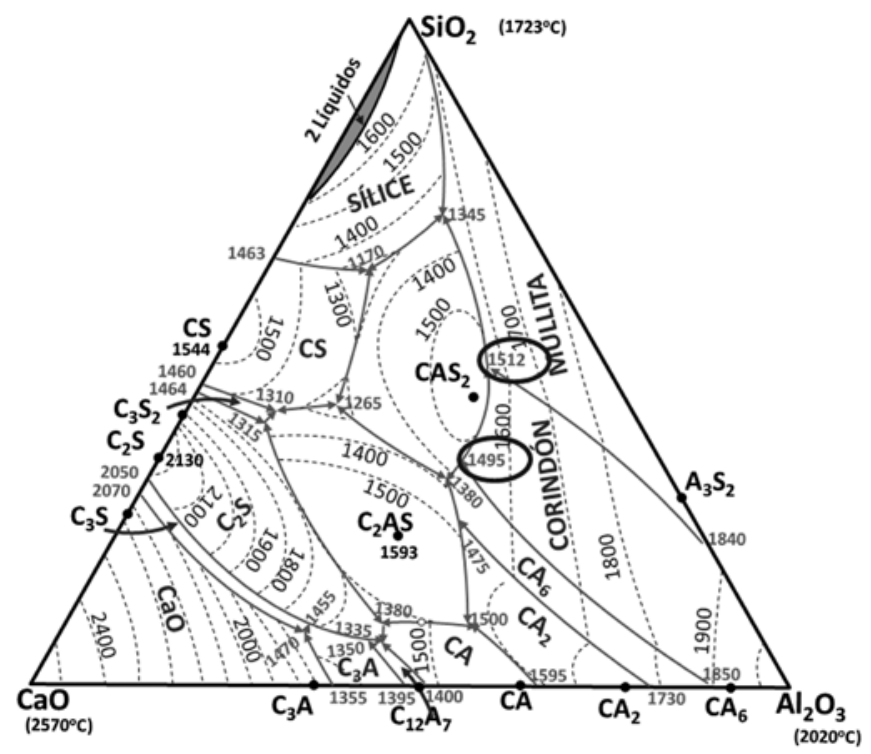

Figura 13. Sistema $\mathrm{Al}_{2} \mathrm{O}_{3}-\mathrm{SiO}_{2}-\mathrm{CaO}[26,24]$. Figura redibujada por $\mathrm{S}$. De Aza [4]. Comparar con la Figura 12. 
aluminatos cálcicos (CAC) con el $71 \%$ u $80 \%$ en peso de alúmina, espinela, o magnesia, y alúmina, como productos de partida [48, 49 , 50]. La composición de estas deberá estar localizada en el triángulo de compatibilidad en estado sólido: $\mathrm{Al}_{2} \mathrm{O}_{3^{\prime}}, \mathrm{MgAl}_{2} \mathrm{O}_{4}$ solución sólida y $\mathrm{CaAl}_{12} \mathrm{O}_{19}$. Dentro del campo primario de cristalización del $\mathrm{Al}_{2} \mathrm{O}_{3}$ (Figura 14).

En las matrices así diseñadas la primera formación de fase líquida tendrá lugar a $1.850^{\circ} \mathrm{C}$, punto invariante peritéctico del subsistema: $\mathrm{Al}_{2} \mathrm{O}_{3}-\mathrm{MgAl}_{2} \mathrm{O}_{4}$ solución sólida-CaAl $\mathrm{O}_{12}$ (Figura 14).

Hormigones, formulados de este modo, han sido utilizados en acería secundaria en el fondo de cucharas de transporte de acero dando excelentes resultados [44, 51, 52]. Adicionalmente, los estudios "post-morten", de las muestras tomadas del fondo de las cucharas, mostraron que la secuencia de fases, encontradas en las secciones trasversales en profundidad del refractario, está en concordancia con la información suministrada por el sistema $\mathrm{Al}_{2} \mathrm{O}_{3}-\mathrm{MgO}-\mathrm{CaO}[44,51,52,53,54]$.

Así, en primera cara el $\mathrm{MgAl}_{2} \mathrm{O}_{4}$ es la única fase sólida estable en contacto con la escoria, ver Figura 15 A, debido a su amplio campo de cristalización, tal como se puede apreciar en la Figura 12 y en la 14, en comparación con los pequeños campos de cristalización del $\mathrm{Al}_{2} \mathrm{O}_{3}$ y el $\mathrm{CaAl}_{12} \mathrm{O}_{19}$ lo que justifica la baja solubilidad de la espinela en la escoria [44, 51, 52].

Justamente por detrás de la primera cara, o cara de trabajo, coexisten, como se puede apreciar, $\mathrm{Al}_{2} \mathrm{O}_{3^{\prime}} \mathrm{MgAl}_{2} \mathrm{O}_{4^{\prime}} \mathrm{CaAl}_{12} \mathrm{O}_{19}$ y una pequeña proporción de fase líquida de acuerdo con el sistema. Al mismo tiempo, debido a que la espinela acepta en su estructura, en solución sólida, una gran cantidad de iones bivalentes y trivalentes (por ejemplo: $\mathrm{Mn}^{2+}, \mathrm{Fe}^{2+}, \mathrm{Fe}^{3+}$ ) [44, 51, 52], la composición de la escoria, que penetra a través de la matriz, cambia haciéndose muy rica en $\mathrm{CaO}$, y al penetrar hacia el interior del material reacciona con el $\mathrm{Al}_{2} \mathrm{O}_{3^{\prime}}$ dando lugar a la formación de un microestructura formada por un entrecruzamiento de cristales de $\mathrm{CaAl}_{12} \mathrm{O}_{19}$ unidos directamente a los cristales de $\mathrm{Al}_{2} \mathrm{O}_{3^{\prime}}$, evitando la penetración de la escoria hacia el interior del material [44, 51, 52]. Figura 15 B.

Los resultados expuestos justifican una vez más la utilización de los diagramas de equilibrio de fases tanto en el diseño de nuevos materiales, como para explicar su comportamiento en servicio.

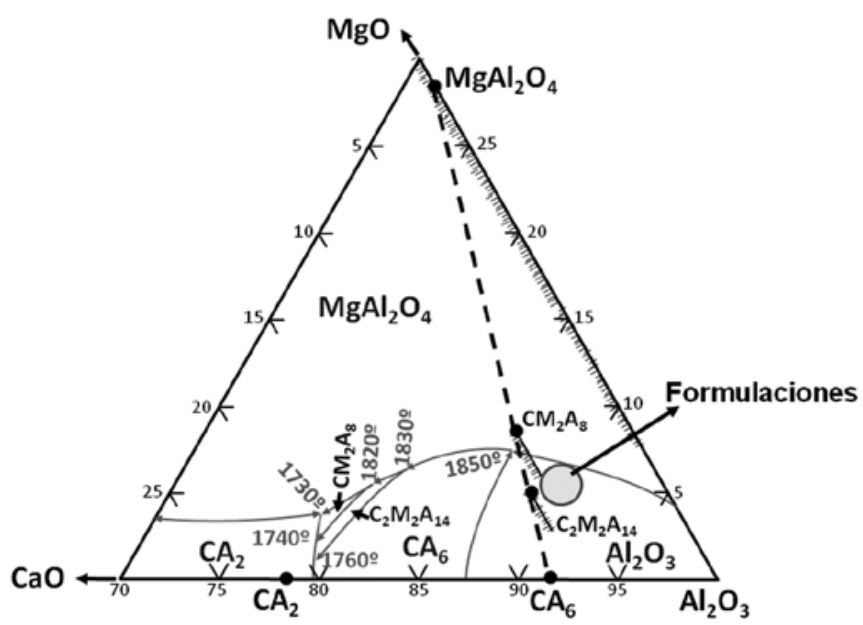

Figura 14. Región de alto contenido en $\mathrm{Al}_{2} \mathrm{O}_{3}$ del sistema ternario $\mathrm{Al}_{2} \mathrm{O}_{3}-\mathrm{MgO}-\mathrm{CaO}$ según A.H. De Aza et al. [45, 46, 44, 24].
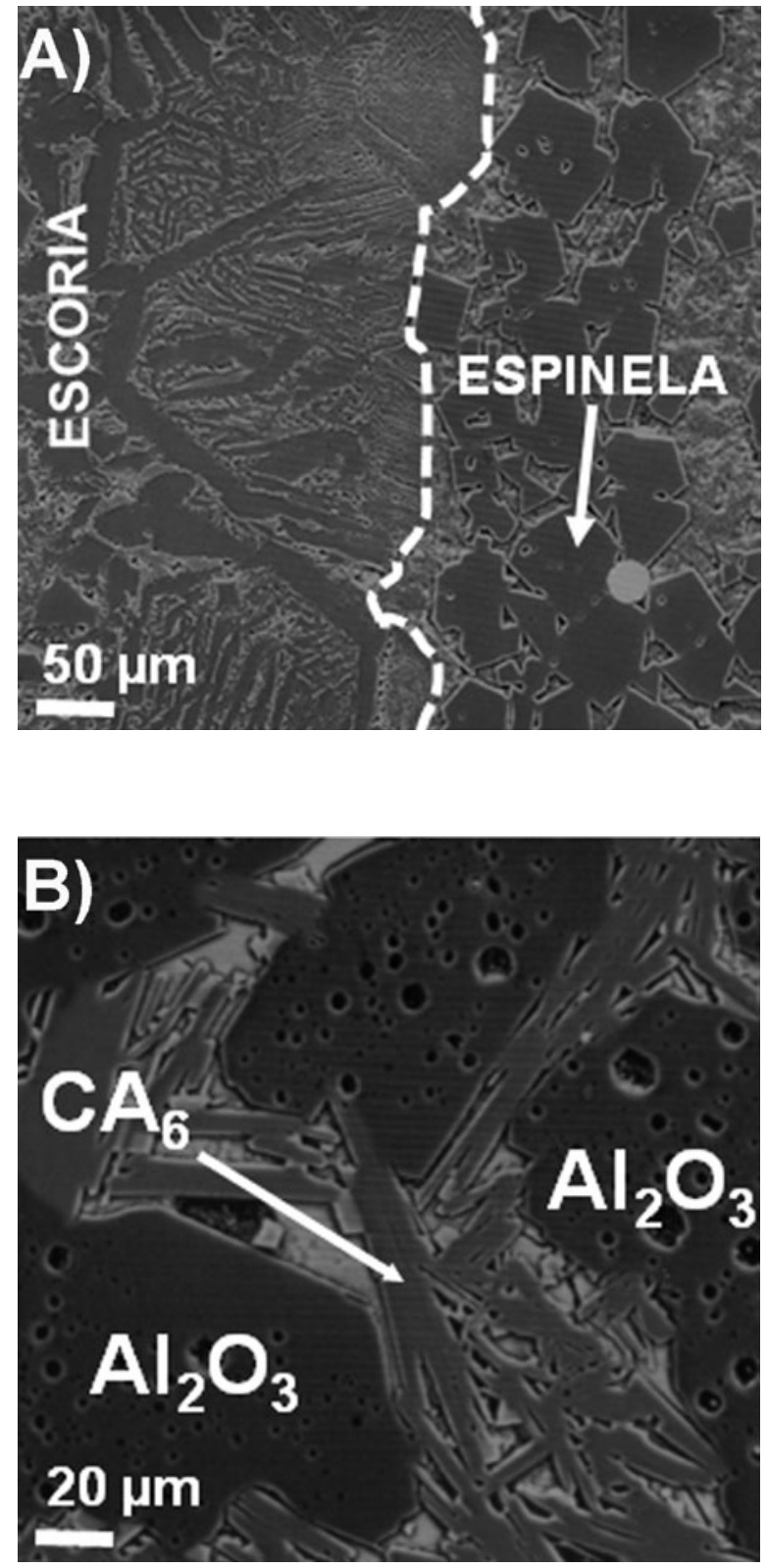

Figura 15. Imágenes obtenidas mediante Microscopía Electrónica de Barrido (MEB). Muestra atacada con ácido HF diluido al $10 \%$ en volumen durante $15 \mathrm{~s}$. En las microestructuras se indican las distintas fases observadas. A) Interfase escoria-refractario. Se confirmó la presencia de espínela $\left(\mathrm{MgAl}_{2} \mathrm{O}_{4}\right)$ como única fase estable en la interfase escoria/refractario. Se pueden visualizar desvitrificaciones de gehlenita $\left(\mathrm{Ca}_{2} \mathrm{Al}_{2} \mathrm{SiO}_{7}\right)$ y merwinita $\left(\mathrm{Ca}_{3} \mathrm{MgSi}_{2} \mathrm{O}_{8}\right)$ en la escoria. Igualmente, se confirmó la presencia de hierro metálico en forma de pequeñas gotas redondeadas y dispersas [51]. B) A partir de $16 \mathrm{~mm}$ de profundidad se observó la presencia de cristales de hexaluminato cálcico $\left(\mathrm{CaAl}_{12} \mathrm{O}_{19}\right)$, con una elevada razón de esbeltez (longitud/anchura), formando puentes entre los granos grandes de alúmina tabular (2-4 $\mathrm{mm})$ [51]. 


\section{NUEVOS CEMENTOS REFRACTARIOS DE ALUMINATOS CÁLCICOS CONTENIENDO ESPINELA}

Para finalizar se muestra un ejemplo enfocado en el diseño de nuevos cementos refractarios de aluminatos cálcicos conteniendo espinela $[18,55]$.

La utilización de estos cementos en la formulación de los hormigones de alta alúmina, evitaría emplear adiciones de $\mathrm{MgO}$ y $\mathrm{Al}_{2} \mathrm{O}_{3}$ calcinada en la matriz para generar el $\mathrm{MgAl}_{2} \mathrm{O}_{4^{\prime}}$ evitando así los problemas que suelen conllevar en la manufactura [48, 49], o bien emplear adiciones de $\mathrm{MgAl}_{2} \mathrm{O}_{4}$ sintéticos, con el elevado costo que acarrean [44].

Para ello se recurrirá nuevamente al sistema ternario: $\mathrm{Al}_{2} \mathrm{O}_{3}-\mathrm{CaO}-\mathrm{MgO}[45,46,47]$ de la Figura 12.

Como se puede apreciar, los campos primarios de cristalización del $\mathrm{MgAl}_{2} \mathrm{O}_{4^{\prime}}$ del monoaluminato cálcico $\left(\mathrm{CaAl}_{2} \mathrm{O}_{4}\right.$ ó $\mathrm{CaO} \cdot \mathrm{Al}_{2} \mathrm{O}_{3}$ ó CA $)$ y del dialuminato cálcico $\left(\mathrm{CaAl}_{4} \mathrm{O}_{7}\right.$ ó $\mathrm{CaO} \cdot 2 \mathrm{Al}_{2} \mathrm{O}_{3}$ ó $\mathrm{CA}_{2}$ ) convergen en el punto invariante

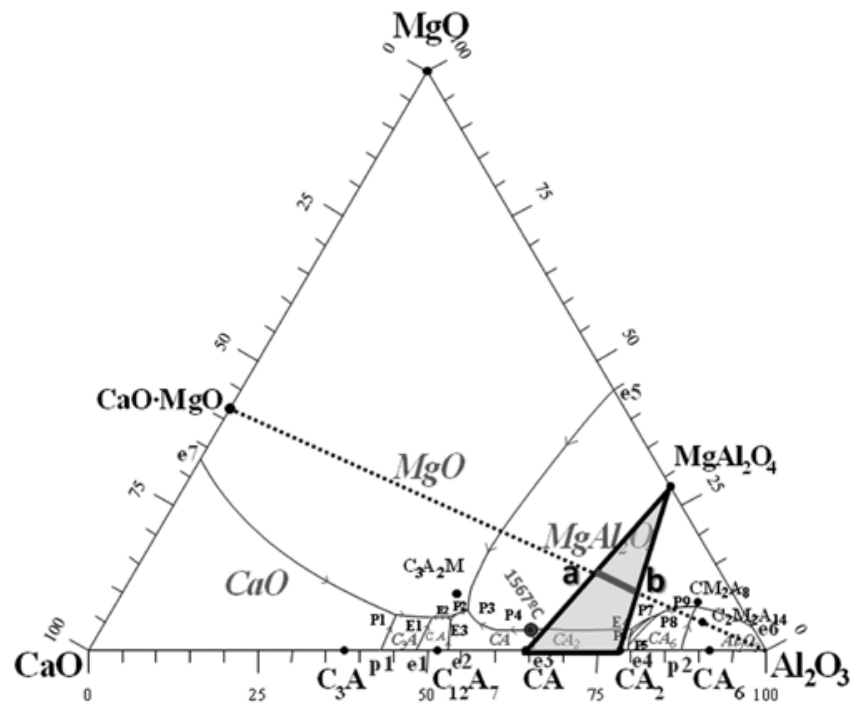

Figura 16. Sistema ternario: $\mathrm{Al}_{2} \mathrm{O}_{3}-\mathrm{CaO}-\mathrm{MgO}[45,46,47,24]$. La figura ilustra el procedimiento desarrollado para obtener nuevos Cementos de Aluminatos Cálcicos $(\mathrm{CAC})$ conteniendo $\mathrm{MgAl}_{2} \mathrm{O}_{4}$ por reacción de $\mathrm{MgCa}\left(\mathrm{CO}_{3}\right)_{2}$ y $\mathrm{Al}_{2} \mathrm{O}_{3}[18,55]$.

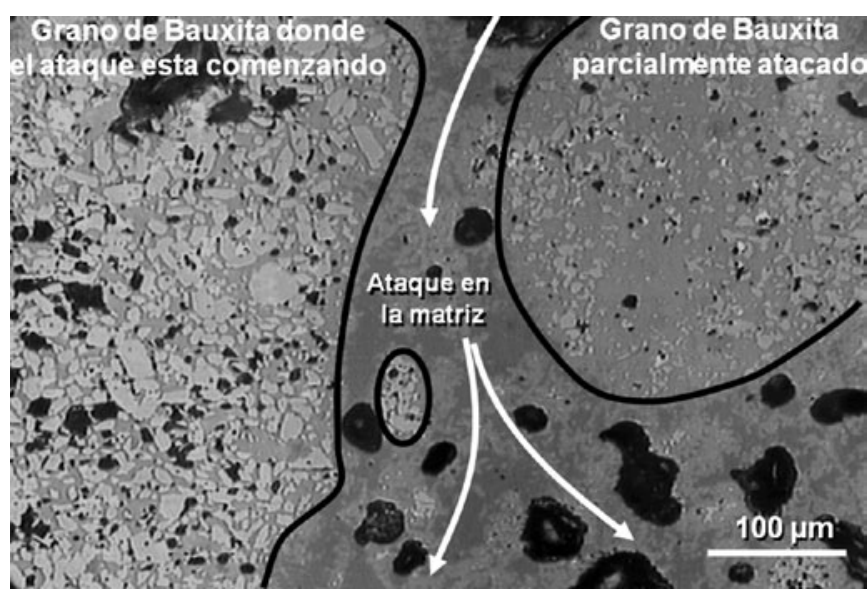

Figura 17. Imagen obtenida mediante de Microscopía Electrónica de Barrido (MEB). Se muestra la microestructura de un hormigón aluminoso empleado en una central térmica. Consultar texto para mayores detalles. peritéctico de $1.567^{\circ} \mathrm{C}$, lo que indica que las tres fases son compatibles en estado sólido por debajo de esa temperatura formando un subsistema ternario. Figura 16.

Cualquier composición dentro de dicho subsistema estará constituida en estado sólido por las mencionadas fases. Esto abre la oportunidad de desarrollar nuevos cementos de aluminatos cálcicos $\left(\mathrm{el}^{\mathrm{CaAl}} \mathrm{O}_{4}\right.$ y el $\mathrm{CaAl}_{4} \mathrm{O}_{7}$ son los constituyentes mayoritarios de los cementos de aluminatos cálcicos) [56, 57, 58 ] conteniendo $\mathrm{MgAl}_{2} \mathrm{O}_{4}$ por reacción de $\mathrm{MgCa}\left(\mathrm{CO}_{3}\right)_{2}$, dolomita, y $\mathrm{Al}_{2} \mathrm{O}_{3}$ en el rango de composiciones del segmento "a-b", según se indica en la Figura 16 [55].

En el caso de utilizar una dolomita estequiométrica, la ecuación que gobierna las diferentes composiciones de dichos cementos, en la región "a-b", es:

$$
\begin{gathered}
(1+y) \mathrm{Al}_{2} \mathrm{O}_{3}+[0,5 \mathrm{MgO}+0,5 \mathrm{CaO}] \\
=(0,5-\mathrm{y}) \mathrm{CaAl}_{2} \mathrm{O}_{4}+\mathrm{y} \mathrm{CaAl}_{4} \mathrm{O}_{7}+0,5 \mathrm{MgAl}_{2} \mathrm{O}_{4}
\end{gathered}
$$

donde $0 \leq \mathrm{y} \leq 0,5$

Siendo posible regular las proporciones de las fases mencionadas, dependiendo del valor que se de a " $y$ ". Por ejemplo, para un valor de $\mathrm{y}=0,075$ la composición de dicho cemento será: $42,56 \% \mathrm{CaAl}_{2} \mathrm{O}_{4^{\prime}}, 12,36 \% \mathrm{CaAl}_{4} \mathrm{O}_{7^{\prime}}, 45,08 \%$ $\mathrm{MgAl}_{2} \mathrm{O}_{4}$.

En el caso de utilizar una dolomita no estequiométrica, la ecuación es:

$$
\begin{gathered}
(1+y) \mathrm{Al}_{2} \mathrm{O}_{3}+[(1-x) \mathrm{MgO}+\mathrm{x} \mathrm{CaO}]= \\
(\mathrm{x}-\mathrm{y}) \mathrm{CaAl}_{2} \mathrm{O}_{4}+\mathrm{y} \mathrm{CaAl}_{4} \mathrm{O}_{7}+(1-\mathrm{x}) \mathrm{MgAl}_{2} \mathrm{O}_{4} \\
\text { donde } 0 \leq \mathrm{x} \leq 1 \mathrm{y} 0 \leq \mathrm{y} \leq \mathrm{x}
\end{gathered}
$$

Toda la información referente a la obtención de dichos cementos puede ser consultada en el trabajo publicado a tal efecto [18] y en la patente registrada a tal efecto [55].

Una vez más, se ha mostrado como haciendo un uso apropiado de los diagramas de fases se pueden diseñar nuevos materiales refractarios.

\section{CONSIDERACION FINAL Y CONCLUSIONES}

A lo largo del presente trabajo se han presentado diferentes ejemplos de la aplicación de los diagramas de equilibrio de fases a la fabricación, diseño y comportamiento en servicio de diversos materiales refractarios. En cuanto a este último punto, es necesario hacer una observación importante.

En el comportamiento de los materiales refractarios en servicio, debe tenerse en cuenta que el ataque suele tener lugar preferentemente a través de la matriz de los mismos (agregados con un tamaño de partícula menor a $125 \mu \mathrm{m}$ ), es decir, de la fracción más fina del material (que es la de mayor superficie específica). Ver Figura 17. En consecuencia, la información suministrada por los diagramas de equilibrio de fases apropiados debe aplicarse a la composición de dichas matrices y no a la composición total del material. De lo contrario se podrá incurrir en graves errores a la hora de predecir o estudiar el comportamiento de los materiales y / o a la hora de introducir mejoras o diseñar nuevos materiales. 
A modo de conclusiones de puede decir que:

A lo largo del artículo se ha pretendido hacer hincapié en la importancia de los diagramas de equilibrio de fases como una herramienta científica para mejorar y/o diseñar nuevos materiales refractarios.

La intención principal ha sido resaltar cómo, a través de un uso adecuado de los diagramas de equilibrio de fases, es posible obtener una información muy valiosa para el diseño, manufactura y comportamiento de los materiales refractarios.

El uso de la información publicada, hoy en día, sobre los distintos diagramas de equilibrio de fases, puede reducir el número de los costosos experimentos necesarios para diseñar nuevos materiales refractarios así como para evaluar sus comportamientos a alta temperatura.

El establecimiento de un diagrama de equilibrio de fases es un trabajo lento y muy laborioso y su utilidad en el progreso de la tecnología refractaria no ha sido siempre bien reconocida.

\section{NOTA DE LOS AUTORES}

Tanto en el campo de los diagramas de equilibrio de fases como en el de los materiales refractarios el Profesor Salvador De Aza colaboró y trabajó con un gran número de científicos, técnicos, estudiantes, etc. y por tanto sería imposible recoger a todos ellos aquí. Somos conscientes de que a una gran mayoría de ellos les hubiera gustado participar en la elaboración de este artículo en homenaje a su memoria. Sirvan estas líneas para reconocer la labor de todos ellos, que esperamos se vean en cierta medida representados por los autores de este articulo, que, sin la menor duda, recoge un simple grano de arena de la dilatada labor realizada en el campo por Salvador De Aza.

A.H. De Aza, P. Pena y A. Caballero en calidad de coautores del presente artículo quisieran hacer constar que el mismo ha sido escrito durante el mes de Octubre de 2011, tras el fallecimiento de Profesor Salvador De Aza, y está basado, fundamentalmente, en su última conferencia plenaria [1].

Aunque,... Profesor, maestro, compañero, amigo y padre (A.H. De Aza) no has podido participar en su elaboración, que sin duda hubieras mejorado (como siempre hacías), pensamos que has de figurar como uno de los autores del mismo. Esperando que allá donde estés, queridísimo Salvador (padre), el resultado final sea de tu agrado. Gracias por tus enseñanzas, motivación, aliento y tantas y tantas cosas que no podemos reflejar aquí y ahora en el papel...Te añoramos,... la semilla que has sembrado siempre estará con nosotros...

\section{AGRADECIMIENTOS}

Los autores agradecen la financiación recibida por el Ministerio de Ciencia e Innovación (MICINN) de España: TRACE PET2008_0114 y MAT2010-17753.

\section{REFERENCIAS}

(1) S. De Aza, "Los diagramas de equilibrio de fases como una herramienta para el diseño y uso de los materiales refractarios" Conferencia Invitada AVR-I-1, 28 Octubre 2010. 50 Congreso de la Sociedad Española de Cerámica y Vidrio Madrid 27-29 Octubre, 2010.
(2) A.H. De Aza, "Phase equilibrium diagrams as a tool for the design and use of refractories". Invited Lecture. Session: CL-1. June 6. CIMTEC 2010. 12th International Ceramics Congress. Montecatini Terme (Italia). June 6-11, 2010.

(3) http:/ / www.rae.es/rae.html

(4) S. De Aza, "Introducción a los materiales refractarios. Curso sobre materiales refractarios (3-8 de junio de 2007). Organizado por el Instituto de Cerámica y Vidrio-CSIC (A. Caballero y A. H. De Aza) y La Asociación Nacional de Fabricantes de Refractarios Españoles (ANFRE), con la colaboración de La Sociedad Española de Cerámica y Vidrio (SECV). 2007.

(5) Refractories, in Section 12 (Application for traditional Ceramics) of Engineered Materials Handbook Volume 4: Ceramics and Glasses. pp 1217. Volume Chairman: S.J. Schneider. Section Chairman: G. Lewis. Edited by ASM International - The Materials Information Society. U.S.A. ISBN 0-87170-282-7, pp 895-909, 1991.

(6) B. Coope, "An introduction to refractories", Raw Materials for the Refractories Industry, Industrial Minerals, 2nd Edition, pp. 7-13, 1986.

(7) Refractories handbook. Edited by Charles A. Schacht. ISBN: 0-8247-5654-1. Published by Marcel Dekker, Inc., New York, pp 499; 2004.

(8) Refractory engineering. Materials-Design-Construction. 2nd revised and update edition. ISBN: 3-8027-3155-7.Edited by GmbH, Vulkan-Verlag., Essen, Germany, pp 456; 2005.

(9) Ingeniería de refractarios. Materiales - Diseño (c) Construcción. (c) Deustche Gesellschaft Fuerfest-und Schornsteinbau e.V. y Asociación Nacional de Fabricantes de Refractarios de España (ANFRE). Varios Autores. Edición Técnica, $1^{a}$ edición en castellano: E. Criado y A. H. De Aza. ISBN 978-848198-825-3; 456 págs. 2010.

(10) J. W. Mellor, "Some chemical and physical changes in the firing of pottery", J. Soc. Chem. Ind., 26 (8) 375-377, 1907.

(11) J.S. Moya, S. De Aza, "Equilibrium diagrams. A tool for designing new ceramics" in Science of Ceramics 14, 27-40, 1988. Edited by D. Taylor, Published by The Institute of Ceramics (UK). 1988.

(12) S. Zhang, W.E. Lee, "Use of phase diagrams in studies of refractories corrosion" International Materials Reviews 45 (2) 41-58, 2000.

(13) W.E. Lee, B.B. Argent, S. Zhang, "Complex phase equilibria in refractories design and use". J. Am. Ceram. Soc. 85 (12) 2911-2918, 2002.

(14) W.E. Lee, B.B. Argent, H. Sarpoolaky, S. Zhang, "Correlating phase equilibria with refractories microstructural evolution on firing and in service" Trans Tech Publications, Switzerland. Key Engineering Materials Vols. 264-268, pp. 1723-1726, 2004.

(15) S. De Aza, J. Espinosa, E. Criado, M.A. del Rio, “Aplicación de los diagramas de equilibrio en la tecnología de refractarios" Bol. Soc. Esp. Ceram. Vidr., 11 (6) 353-366, 1972.

(16) J. Espinosa, S. De Aza, E. Criado, M.A. del Rio, “Aplicación de los diagramas de fase ternarios a los productos de cerámica blanca Bol. Soc. Esp. Cerám. Vidr., 12 (1) 31-39, 1973.

(17) S. De Aza, "Diagramas de fase" en Propiedades Mecánicas de Sólidos, p.69-83, Edit. R. Márquez, A. Dominguez, O. Ruano, M. Jimenez. Publ. Universidad de Sevilla. 1988.

(18) A.H. De Aza, P. Pena, M. A. Rodriguez, R. Torrecillas, and S. De Aza, "New spinel-containing refractory cements," J. Eur. Ceram. Soc., 23 (5) 737-44, 2003.

(19) J.L. Rodriguez, M.A. Rodriguez, S. De Aza, P. Pena, “Reaction sintering of zircon-dolomite mixtures" J. Eur. Ceram. Soc., 21 (3) 343-354, 2001.

(20) A.H. De Aza, P. Pena, J.S. Moya, "Reactive coating of dolomite on alumina substrates" J. Eur. Ceram. Soc. 17 (7) 935-941, 1997.

(21) J.S. Moya; A.H. De Aza; H.P. Steier, J. Requena, P. Pena, “Reactive coating on alumina substrates - calcium and barium hexaaluminates" Scripta Metallurgica et Materialia 31 (8) 1049-1054, 1994.

(22) M. Flora Barba Martín-Sonseca. “Constitución de los materiales refractarios, efecto de las impurezas" Capítulo IV (pp 50-130) en "Nuevas sistemáticas para el análisis completo de materiales refractarios. Elementos clave". Tesis Doctoral. ICV-CSIC. Universidad Complutense de Madrid. Facultad de Ciencias Químicas. 1986.

(23) B. Phillips, A. Muan, "Phase equilibria in the system $\mathrm{CaO}$ lron Oxide $\mathrm{SiO}$ in air. J. Am. Ceram. Soc. 42 (9) 413-423, 1959.

(24) ACerS-NIST. Phase Equilibria Diagrams. CR-ROM Databese, Version 3.1, and Books, Volume I to XIV. Edited by The American Ceramic Society (1964 - 2005). http://ceramics.org/publications-and-resources/phaseequilibria-diagrams

(25) W. M. Huang, M. Hillert, X. Z. Wang, “Thermodynamic Assessment of the. CaO-MgO-SiO 2 System," Metall. Mater. Trans. A, 26A (9) 2293-2310, 1995.

(26) E.F. Osborn, A. Muan, "System $\mathrm{CaO}-\mathrm{Al} \mathrm{O}-\mathrm{SiO}$; revised and redrawn" in "Phase Equilibrium Diagrams of Oxide Systems," Plate 1, published by the American Ceramic Society and the Edward Orton, Jr., Ceramic Foundation, 1960.

(27) W.A. Deer, R.A. Howie, J. Zussman. An introduction to the rock-forming minerals (2nd Edition). ISBN 978-0-582-30094-1. Pearson Education Limited. Edinburgh Gate. Harlow. Essex CM20 2JE. England. First edition 1996. Second Edition, pp 687, 1992. 
(28) C. S. Hurlbut Jr., C. Klein. Manual de mineralogía de Dana. $3^{\text {a }}$ Edición. Editorial Reverté S.A. Barcelona, España. ISBN - 84-291-4605-9. pp564, 1985.

(29) W.E. Lee, W. M. Rainforth. Refractories, Chapter 8 (pp 452-507) in Ceramic Microstructures Property Control by Processing. Published by Chapman \& Hall, London UK pp590, 1994.

(30) W. Baumgart, in Process mineralogy of ceramic materials. Eds W. Baumgart, A.C. Dunham and G.C. Amstutz. Elsevier, pp. 80-103, 1984.

(31) F.P. Hall, H. Insley, E.M. Levin, H.F. McMurdie, C.R. Robbins, “General Discussion of Phase Diagrams" in Phase Diagrams for Ceramists, Volume I; edited by E.M. Levin, C.R. Robbins and H.F. McMurdie, pages 5-36, 1964. http: / / ceramics.org/wp-content/uploads/2008/12/general_discussions_ phase_diagram.pdf

(32) C.S. Bergeron and S.H. Risbud. Introduction to phase equilibria in ceramics. Edited by The American Ceramic Society Inc. pp 158, 1984.

(33) W. F. Ford, The Effect of Heat on Ceramics. Fig 51, page 93. Edited by Maclaren and Sons LTD., London England, pp 185, 1967.

(34) S. De Aza, C. Richmond, J. White, "Compatibility Relationships of Periclase in the System CaO-MgO- $\mathrm{ZrO}_{2}-\mathrm{SiO}_{2}$ " Trans. J. Br. Ceram. Soc., 73 (4) 109-116, 1974.

(35) J.L. Rodríguez Galicia. Tesis Doctoral. “Diseño y desarrollo de materiales de magnesia con matriz de circonato cálcico y silicato dicálcicos para aplicaciones en hornos de cemento". ICV-CSIC. Universidad Autónoma de Madrid. Mayo 2001.

(36) J.L. Rodriguez-Galicia, A.H. De Aza, J.C. Rendon-Angeles, P. Pena. "The Mechanism of corrosion of $\mathrm{MgO}-\mathrm{CaZrO}_{3}$-calcium silicate materials by cement clinker" J. Eur. Ceram. Soc. 27 (1) 79-89, 2007.

(37) J.L. Rodriguez, A.H. De Aza, S. De Aza, P. Pena, J. Campo, P. Convert, $\mathrm{X}$. Turrillas, "Study of zircon-dolomite reactions monitored by neutron thermodiffractometry" J. Solid State Chem. 166 (2) 426-433, 2002.

(38) Sara Serena Palomares. Tesis Doctoral. "Modelización termodinámica y cálculo del diagrama de equilibrio de fases $\mathrm{ZrO}_{2}-\mathrm{CaO}-\mathrm{MgO}$. Aplicación al diseño y obtención de materiales de $\mathrm{MgO}-\mathrm{CaZrO}_{3}{ }^{\prime \prime}$. ICV-CSIC. Universidad Autónoma de Madrid. Marzo 2002.

(39) S. Serena, A. Caballero; M.A. Sainz, P. Convert, J. Campo, X. Turrillas, "Neutron thermodiffractometry study of calcium zirconate/magnesium oxide formation in the $\mathrm{ZrO}_{2}-\mathrm{CaO}-\mathrm{MgO}$ system" J. Am. Ceram. Soc. 87 (9) 1706-1713, 2004.

(40) P. Pena, A. H. De Aza, A. Caballero. "Residuos refractarios con elevados contenidos en cromo. Proceeding", II Congreso Nacional del Medio Ambiente. Editado por Colegio Oficial de Físicos. Vol.4, pp. 73-80, 1994.

(41) D. J. Bray, "Toxicity of chromium compounds formed in refractories". Ceramic Bull, 64, 1012-016, 1985.

(42) W. E. Lee, W. Vieira, S. Zhang, K. Ghanbari Ahari, H. Sarpoolaky, C. Parr, "Castable refractory concretes," Int. Mater. Rev., 46 (3) 145-67, 2001.

(43) S. Banerjee, T. Abraham, "Changing the face of the global refractories industry," Am. Ceram. Soc. Bull., 78 (5) 55-8, 1999.

(44) A.H. De Aza. Tesis Doctoral. "Diseño y desarrollo de materiales de alta alúmina con matrices de espinela y hexaluminato cálcico". ICV-CSIC. Universidad Autónoma de Madrid. Octubre 1997.
(45) A. H. De Aza, P. Pena, S. De Aza, "The System $\mathrm{Al}_{2} \mathrm{O}-\mathrm{MgO}-\mathrm{CaO}$. Part I Primary phase field of spinel in the subsystem $\mathrm{MgAl}_{2} \mathrm{O}_{4}-\mathrm{CaAl}_{4} \mathrm{O}_{7}-\mathrm{CaO}-$ MgO," J. Am. Ceram. Soc., 82 (8) 2193-203, 1999.

(46) A. H. De Aza, P. Pena, J. E. Iglesias, S. De Aza, "The System $\mathrm{Al}_{2} \mathrm{O}_{3}-\mathrm{MgO}$ $\mathrm{CaO}$. Part II. Relationship in the subsystem $\mathrm{Al}_{2} \mathrm{O}_{3}-\mathrm{MgAl}_{2} \mathrm{O}_{4}-\mathrm{CaAl}_{4} \mathrm{O}_{7}^{\prime \prime} \mathrm{J}$. Am. Ceram. Soc., 83 (4) 919-27, 2000.

(47) T. Duran, S. Serena, P. Pena, A. Caballero, S. De Aza, A. H. De Aza, "Experimental Establishment of the $\mathrm{CaAl}_{2} \mathrm{O}_{4}-\mathrm{MgO}$ and $\mathrm{CaAl}_{4} \mathrm{O}_{7}-\mathrm{MgO}$. Isoplethal sections within the $\mathrm{Al}_{2} \mathrm{O}_{3}-\mathrm{MgO}-\mathrm{CaO}$ ternary system," J. Am. Ceram. Soc., 91 (2) 535-43, 2008.

(48) T. Durán, P. Pena, S. De Aza, J. Gómez-Millán, M. Alvarez, and A.H. De Aza, "Interactions in calcium aluminate cement (CAC)-based castables containing magnesia. Part I: Hydration-Dehydration behavior of $\mathrm{MgO}$ in the absence of CAC" J. Am. Ceram. Soc. 94 (3) 902-908, 2011.

(49) T. Durán, P. Pena, S. De Aza, J. Gómez-Millán, M. Alvarez, and A.H. De Aza, "Interactions in calcium aluminate cement (CAC)-based castables containing magnesia-Part II: Hydration-Dehydration behavior of CAC and their mixtures with dead-burned and reactive-grade $\mathrm{MgO}^{\prime \prime} \mathrm{J}$. Am. Ceram. Soc. 94 (3) 909-17, 2011.

(50) L.A. Diaz, R. Torrecillas, A.H. De Aza, P. Pena, S. De Aza " Aluminarich refractory concretes with added spinel, periclase and dolomite: A comparative study of their microstructural evolution with temperature" J. Eur. Ceram. Soc. 25 (9) 1499-1506, 2005.

(51) A. H. De Aza, P. Pena, S. De Aza, M. Raigón, J. Gómez-Millán, C. Domínguez "Estudio post-mortem de un hormigón refractario colocado en la línea de escorias de una cuchara de acería" Bol. Soc. Esp. Ceram. Vidr. 42 (6) 375-8, 2003.

(52) C. Dominguez, M. Raigón, J. Gómez-Millán, F. J. Ferrer, E. Pérez, A. H. De Aza, A. Caballero, S. DeAza, "Bottom working lining improvement in stainless steel ladles", Proceedings of Unified International Technical Conference on Refractories, 7th Biennial Worldwide Congress, UNITECR'2001, Cancun, Mexico, Hosted by ALAFAR, November 4-7, 2001, pp. 289-299, 2001.

(53) L.A. Díaz, R. Torrecillas, A. H. De Aza, P. Pena, “Effect of spinel content on slag sttack resistance of high alumina refractory castables" J. Eur. Ceram. Soc., 27 (16) 4623-31, 2007.

(54) L. A. Diaz, R. Torrecillas, "hot bending strength and creep behaviour at $1000^{\circ}-1400^{\circ} \mathrm{C}$ of high alumina refractory castables with spinel, periclase and dolomite additions" J. Eur. Ceram. Soc., 29 (1) 53-8, 2009.

(55) A. H. De Aza, R. Torrecillas, P. Pena y S. De Aza. Cementos refractarios aluminosos conteniendo espinela y procedimientos de obtención. Patente. Titular: CSIC. $\mathrm{N}^{\circ}$ de solicitud: 00970115. $\mathrm{N}^{\circ}$ de publicación: 2143 369. Países a los que se ha extendido: España. Fecha de concesión: 01/01/2001.

(56) J.M.R. Mercury, A.H. De Aza, P. Pena, “Calcium aluminate cements hydration (Part I)". Bol. Soc. Esp. Ceram. Vidr. 42 (5) 269-276, 2003.

(57) J.M.R. Mercury, A.H. De Aza, P. Pena, "Calcium aluminate cements hydration. Part II: Effect of silica and alumina additions" Bol. Soc. Esp. Ceram. Vidr. 42 (6) 361-368, 2003.

(58) J.M.R. Mercury, X. Turrillas, A.H. De Aza, P. Pena, “Calcium aluminates hydration in presence of amorphous $\mathrm{SiO}_{2}$ at temperatures below $90^{\circ} \mathrm{C}^{\prime \prime} \mathrm{J}$. Solid State Chem. 179 (10) 2988-2997 2006. 